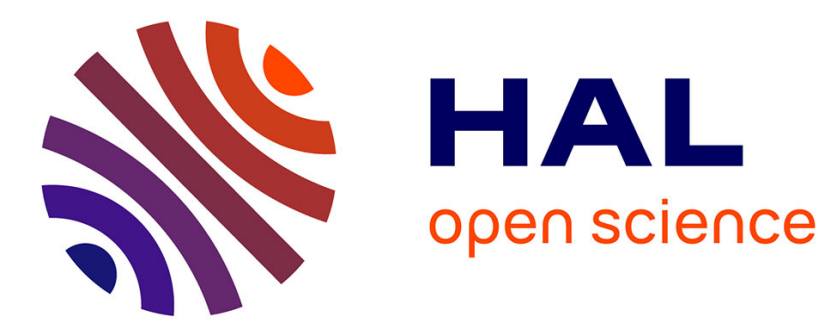

\title{
Tolerance analysis - Form defects modeling and simulation by modal decomposition and optimization
}

Lazhar Homri, Edoh Goka, Guillaume Levasseur, Jean-Yves Dantan

\section{To cite this version:}

Lazhar Homri, Edoh Goka, Guillaume Levasseur, Jean-Yves Dantan. Tolerance analysis - Form defects modeling and simulation by modal decomposition and optimization. Computer-Aided Design, 2017, 91, pp.46-59. 10.1016/j.cad.2017.04.007 . hal-01558235

\section{HAL Id: hal-01558235 \\ https://hal.science/hal-01558235}

Submitted on 7 Jul 2017

HAL is a multi-disciplinary open access archive for the deposit and dissemination of scientific research documents, whether they are published or not. The documents may come from teaching and research institutions in France or abroad, or from public or private research centers.
L'archive ouverte pluridisciplinaire HAL, est destinée au dépôt et à la diffusion de documents scientifiques de niveau recherche, publiés ou non, émanant des établissements d'enseignement et de recherche français ou étrangers, des laboratoires publics ou privés. 


\title{
Tolerance analysis - Form defects modeling and simulation by modal decomposition and optimization
}

\author{
Lazhar Homri *, Edoh Goka, Guillaume Levasseur, Jean-Yves Dantan \\ LCFC, Arts et Métiers ParisTech, HESAM, Université de Lorraine, 4 rue Augustin Fresnel, 57078 METZ Cedex 3, France
}

\begin{abstract}
A B S T R A C T
Tolerance analysis aims on checking whether specified tolerances enable functional and assembly requirements. The tolerance analysis approaches discussed in literature are generally assumed without the consideration of parts' form defects. This paper presents a new model to consider the form defects in an assembly simulation. A Metric Modal Decomposition (MMD) method is henceforth, developed to model the form defects of various parts in a mechanism. The assemblies including form defects are further assessed using mathematical optimization. The optimization involves two models of surfaces: real model and difference surface-base method, and introduces the concept of signed distance. The optimization algorithms are then compared in terms of time consumption and accuracy. To illustrate the methods and their respective applications, a simplified over-constrained industrial mechanism in three dimensions is also used as a case study.
\end{abstract}

\section{Introduction}

A manufactured product always has different geometric features from the defined nominal characteristics irrespective of the manufacturing processes employed and the materials used. Tolerance analysis aims to subsequently study the influence of such geometric variations within the obtained features on the behavior of a mechanical system by checking if the geometric tolerances of the components ensure the compliance of a mechanical system in terms of functional requirements. Tolerances analysis also has an impact on all the stages of a product lifecycle such as designing, process planning, etc. and allows to improve the product quality by reducing the associated cost and ensuring reliability [1,2].

Traditionally, there are two major types of tolerance modeling: statistical estimation and worst-case estimation. By applying the statistical analysis (e.g., based on Monte-Carlo simulation), the tolerances can ensure acceptability of a certain large number of assemblies [3,4]. The worst-case methods, on the other hand, generate a functional assembly from the combination of dimensions and tolerances of individual components. Therefore, the tolerances which satisfy the worst case method ensure $100 \%$ acceptability of the assemblies [5].
Moreover, tolerancing activities, when simulating geometric deviations, are generally based on the hypothesis that parts are ideals and surfaces have no form defects [4-7]. The consideration of parts' form defects is integral to the tolerancing activities and hence, make it significant to highlight its impacts on the assembly probability estimation in the product quality and cost assessment [8,9]. Grandjean et al. [10] mentioned that the non-consideration of form errors could lead to noncompliant assemblies even if all parts respect the geometrical specifications. Generally, the need to predict and manage all deviations is an important issue in product design, tolerance synthesis and assembly simulation [11]. Globally, there are three main issues in tolerance analysis: geometrical defects modeling, geometrical behavior modeling and technical solutions determining. Each of the issues is explained in the following paragraphs:

\section{Geometrical defects modeling}

For geometrical defects modeling, real surfaces derived from a manufacturing process, can be modeled by substituted surfaces [12]. A 'substituted surface' is an ideal surface (geometrically perfect) that is not only the same as the nominal surface but also characterizes a specific physical realization. Furthermore, the substituted-surface model is used to simulate geometric defects in a real surface by situation and dimension deviations. 'Situations deviations' can be mathematically represented with the help of the vectors [13], by a small displacement torsor SDT [12], by a matrix [14] or by a metric torsor [15]. Also, the deviations between two surfaces potentially in contact can be formalized based on the 
SDT and substituted models cannot take form defects into account when characterizing geometrical defects.

Many authors proposed different techniques to geometrically model the defects. For example, Morière [16] proposed to use polynomial functions of degree '2' to model surfaces with defects. Merkley [17] also developed the use of random Bezier curves to model the geometrical defects wherein shape deviations were parameterized by constraining the displacement of the control points of Bezier curves. Lagrange polynomials (or splines) and Bezier surfaces including parts' form defects are traditionally considered in Computer Aided Design [18]. Franciosa et al. [19] presented a morphing mesh-based approach to generate variational shapes according to a small set of points in the nominal geometries of parts. From the mesh model of parts, the associated nodes were moved by applying a morphing procedure. Deviation points, called control points, then defined the concerning local deformations of the surfaces of the parts.

Another concept for modeling parts deviations is the 'Skin Model' which was introduced by Ballu and Mathieu to provide a global representation of the parts' surfaces and acts to express geometric specifications $[20,21]$. The concept stemmed from the theoretical foundations of Geometrical Product Specification (GPS) and aimed to enrich the nominal idealized geometry considering physical shapes. The Skin model also represents the interface of the part with its environment [22]. It has been used in some recent works by Schleich et al. [11,23], for the assembly simulation of over-constrained systems. Skin Model shapes were used by Schleich et al. [24] as a finite model to illustrate the specific realizations of the skin model in conjunction with geometric deviations being resulted from manufacturing and assembly processes. In short, the skin model shapes are specific outcomes of the skin model employing discrete geometry representation schemes, such as point clouds and surface meshes. Based on this concept, an assembly simulation approach integrating point-based Skin Model Shapes, along with mathematical constrained optimization and difference surface definition, are proposed [23].

In addition to the above discussed issue of modeling geometrical part deviation, some recent works which deal with the decomposition and reconstruction of parts' form defects of simple geometry (e.g., cylinder, plane), have also been developed. Discrete-Cosine-Transformation based decomposition method was proposed by Huang et al. [25] and decomposed the defect into a series of independent modes where each mode can represent various manufacturing defect patterns. For example, the deformation mode can represent part distortion during stamping operations. The method has been adopted by Lecompte et al. [26] to predict the form defects of plane surface as a sum of individual technological defects while the orientation and position defects were not taken into account. Moreover, it was extracted that this method worked well just on rectangular-based surface. Fourier series decomposition method based on Discrete Fourier Transform (DFT) was then introduced by Raja and Radhakrishnan [27] to model form defects which could be applied in most cases to discrete objects. The shape of the geometry can be thus be reconstructed by DFT inverse. Henke et al. [28] also used the Tchebychef Fourier Series model to describe the forms of a cylinder and identify specific types of error shapes. A model of eigen-shapes derived from a Principal Component Analysis (PCA) was also proposed considering the information from measurements to simulate shapes [29].

Samper et al. [30] developed the Discrete Modal Decomposition (DMD) which was initiated on the correlation between sound vibration of a bell and geometric defects. The DMD is based on the natural mode shapes of a discretized feature. The modes are generated by a finite element mesh and a modal basis can then be built. Each defect is characterized as a combination of elementary modes that model the geometric shape. To achieve that, the initial

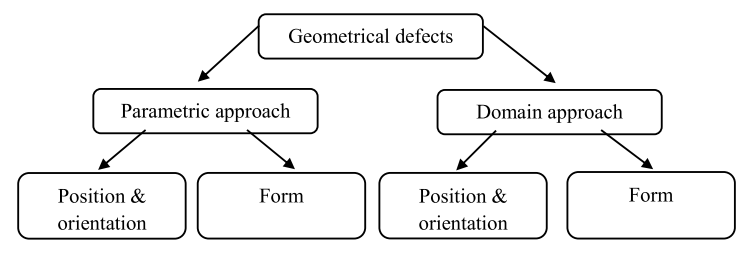

Fig. 1. Geometrical defects modeling.

geometric element is discretized and transformed into a discrete mechanical structure. The structure is then modeled by a stiffness matrix $\mathbf{K}$ and a mass matrix $\mathbf{M}$. The modes are obtained by solving the differential equation of a conservative system; $\mathbf{M} \ddot{\mathbf{q}}+\mathbf{K q}=0$, introducing the two matrices. Modal rebuilding then provides the surface with form defects and a surface $\mathbf{T}$ can be represented as follows:

$\mathbf{T}=\sum_{i=1}^{n} \lambda_{i} Q_{i}$

where $n$ defines the number of modes, $Q_{i}$ are the elementary modes characterizing the surface with form defects, and $\lambda_{i}$ are the amplitudes of modes. The method requires a large amount of measurement data and a particular modal solver.

Fig. 1 depicts a classification scheme of the geometrical defects modeling detailed in this section. The substituted surface dealt with position and orientation defects and is generally considered part of the domain approaches. Domain approaches are defined when situations are handled with mathematical constraints or equations. Skin model, DCT, DFT and DMD take form defects of parts into consideration especially when developing parametric approaches and this was implemented in various commercial tools for tolerance analysis.

\section{Geometrical behavior modeling}

Behavior modeling involves the definition of the mathematical models to characterize the system behavior with deviations by knowing how features of systems interact. The mathematical formulations depend on a diagram (called Joint Graph) describing the features, the links between them (with or without gaps), and the functional conditions to specify the global topological structure of the mechanical systems through dimensional chains [31]. Moreover, the mathematical formulations are defined by: (i) equations which constraint part deviations, gaps and functional characteristics; (ii) inequalities which constraint functional requirements, and (iii) inequalities and equations which constraint gaps. Here, the equations define the relations of displacements in the different loops of the joint graph, the relations of displacements represent the linear compatibility constraints between deviations and gaps in different loops, while the inequalities and equations define the interface constraints that characterize the non-interferences between surfaces that are nominally in contact with each other [32-36]. Nevertheless, considering positional deviations in a 3-dimensional context could lead to highly nonlinear functions which then have to be linearized piecewise [7,37].

Functional requirements which are represented by inequalities limit the displacements (orientation and location) between surfaces in functional condition. To ensure these requirements, all inequalities of non-interference and compatibility equations must be respected. Recently, Lê et al. [38] proposed the concept of a gap hull and the introduction of difference surface to study the behavior of a planar joint when surfaces are with form defects. Some other studies give matting solutions for circular [39] or prismatic joints [8]. 
Technical solutions and analysis approaches

To simulate geometrical variations of a surface, within a tolerance zone or non-interference between surfaces potentially in contact, several works have dealt with methods based on the handling of mathematical formulations and relations based on the kinematic chains (Minkowski sum and intersection) of displacements. The set of these mathematical relations can be deduced from the topological loops of the assembly graph characterizing the mechanical system [31]. Giordano et al. [40] introduced the clearance and deviation domains which are 3D spaces of displacements defined through the SDT of mating surfaces or specified surfaces on the basis of which the geometric specifications (position and orientation) are considered. A similar concept on deviation and clearance spaces was proposed by Fleming [41,42]. Moreover, Morse and Zou [43] proposed the GapSpace Model for two-dimensional assemblies. The model used gaps to describe the possible mating (zero gaps) or clearance (non-zero gaps) conditions between features within the assemblies. Fitting conditions and mating conditions were also arranged in an assembly graph described by inequalities that introduced the gaps. Davidson and Shah [34] further proposed the T-Maps ${ }^{\circledR}$ model. The model enabled a 3D simulation of all variations of features (i.e., size, orientation and form) [44,45] and links [35]. Homri et al. [7] also developed polytopes method to simulate variations in the overconstrained mechanisms. These mathematical representations of tolerances allowed computing accumulation of the tolerances by Minkowski sums or intersections and the operations depended on the kinematic chains. Based on the definition of the probability of defects due to the manufacturing imperfections by different techniques which aim to provide suitable quality level of designed mechanical systems at lower costs, some tolerance analysis models have also been proposed which are based on the statistical analysis method $[4,5,46,47]$.

As the product behavior is disturbed by component variations and gaps between components, tolerance analysis methods should consider not only the changes in components as random values (i.e., income manufacturing) but also the worst possible combination of gaps. These considerations vary depending on the nature of possible products in behavior modeling. Substituted surface model was often considered to characterize such geometrical deviations and gaps. The relationship between the input parameters (deviations $\mathbf{x}$ and gaps $\mathbf{g}$ without interference between parts) and the response $\mathbf{y}$, of the mechanical assembly can be expressed by a function $f$ such that $\mathbf{y}=f(\mathbf{x}, \mathbf{g})$ where $\mathbf{x}=\left(x_{1}, \ldots, x_{n}\right)$ are continuous random variables, typically derived from the drawing dimensions, and $\mathbf{g}=\left(g_{1}, \ldots, g_{n}\right)$ are the values for the gaps [6,45]. The goal of such approaches is to estimate the probability of system conformity by simulation based methods (e.g., Monte Carlo Simulation) or point based method (e.g., FORM system), and optimization $[36,37]$. Further investigations for the computational problem of the function $f$ have been proposed by Turner and Nigam [6], Guilford and Turner [48] and Zhang et al. [49]. Whitney [50] also suggested fitting a multi-dimensional Gaussian Probability Density Function (PDF) to the multi-dimensional possibilities that are permitted by a tolerance specification.

Pertaining to technical solutions, a rich overview of tolerance analysis models of over-constrained mechanisms is also given in $[3,6,7,34,38,41,51-57]$. Globally, the main shortcomings of the tolerance analysis approaches are their insufficient consideration of parts' form defects in the respective systems.

In light of the in-depth literature survey discussed in the preceding paragraphs, the present work highlighted in this paper incorporated a Metric Modal Decomposition (MMD) approach to generate form defects of a cylindrical surface considering the deviations occurring in the nodes of a surface mesh. Each deviation, caused by a "perturbation" function, determines an individual defect mode. Moreover, the obtained form defect is defined by a combination of the individual modes and the overall method is based on geometrical aspects detaching any possible link to Finite Element (FE) modeling, Statistical Shapes Analysis or Principal Component Analysis (PCA). The rest of the paper is divided as follows: Section 2 summarizes the proposed methodology; Section 3 presents the development and determination of an assembly simulation via mathematical optimization to include form defects; and finally, Section 4 describes the application of the proposed methodology on an example of an over-constrained mechanism.

\section{Form defects modeling by Metric Modal Decomposition (MMD)}

\subsection{Methodology}

Inspired from the Discrete Modal Decomposition [30], a new method to generate form defects of cylinders is proposed in this paper. The method, called MMD is based on the definition of a vector field instead of modal basis $\left(Q_{i}\right)_{i}$ in order to show analytically the modes according to the coordinates of each point in the surface. Each modal vector is defined by the displacements in each node of the mesh. A modal field can be defined in an $(\mathbf{x}, \mathbf{y}, \mathbf{z})$ base by Eq. (2):

$\mathbf{u}(x, y, z)=\left(\begin{array}{l}\mathbf{u}_{x}(x, y, z) \\ \mathbf{u}_{y}(x, y, z) \\ \mathbf{u}_{z}(x, y, z)\end{array}\right)$.

For each point $\mathrm{M}_{0}\left(x_{0}, y_{0}, z_{0}\right)$ in the nominal surface, the corresponding point $\mathrm{M}_{1}\left(x_{1}, y_{1}, z_{1}\right)$ in the non-ideal surface can be defined by Eq. (3):

$$
\left(\begin{array}{l}
x_{1} \\
y_{1} \\
z_{1}
\end{array}\right)=\left(\begin{array}{l}
x_{0} \\
y_{0} \\
z_{0}
\end{array}\right)+\left(\begin{array}{l}
\mathbf{u}_{x}\left(x_{0}, y_{0}, z_{0}\right) \\
\mathbf{u}_{y}\left(x_{0}, y_{0}, z_{0}\right) \\
\mathbf{u}_{z}\left(x_{0}, y_{0}, z_{0}\right)
\end{array}\right) .
$$

For a cylindrical surface, the direction of $\mathbf{u}$ is consistent with the direction of the radius that goes through $\mathrm{M}_{0}$. Therefore, the cylindrical surface including form defects (non-ideal surface) is the superposition of the nominal cylindrical surface and these defects. The vector fields that generate form defects are generally represented through a function, rather than a numerical value. The global modal field $\mathbf{u}$ characterizing the non-ideal surface is defined by the weighted sum of basic modes $\mathbf{u}_{i}$ with amplitudes $\lambda_{i}$ generated randomly as shown in Eq. (4):

$\mathbf{u}=\sum_{i=1}^{n} \lambda_{i} \mathbf{u}_{i}$.

All possible form defects depend generally on the nominal geometries of surfaces. Intrinsic features and degrees of invariance of nominal surfaces are taken into consideration to define the modes. Three classes of modes can be identified:

- Rigid mode $\left(\mathbf{u}_{r}\right)$ : The mode cannot "perturb" the ideal geometry of surfaces. The mode is defined by translation or rotation of surfaces. A cylinder is unchangeable by rigid mode and rigid modes are generally the first natural modes of surfaces.

- Rippled mode $\left(\mathbf{u}_{o}\right)$ : The mode is defined by sinusoidal deviations of the surface perpendicular to the axis of invariance. This mode exists only if surfaces have at least one invariance axis (e.g., axis of a cylinder).

- Mode of section (elliptic mode) $\left(\mathbf{u}_{s}\right)$ : The mode is defined by variations of the intrinsic characteristics using polar coordinates. For a cylinder identified by its axis $\mathbf{z}$ and a radius $r$ with an angle $\theta$ for polar coordinates, an elliptic mode is defined by the variations of the radius $r$ by $\mathbf{z}$ or by $\theta$. 
To better understand and illustrate the methodology, the generation of different modes is applied on a right circular cylinder in the subsequent text.

\subsection{MMD for cylindrical surfaces}

A cylindrical surface is considered in this paper to extend the method to complex geometries. Additionally, application through intrinsic features such as a radius and two degrees of invariance (a rotation and a translation along the axis of revolution), can also be illustrated through the three modes explained above. It is imperative to note that from now on, the $\mathbf{z}$-axis identifies the axis of revolution of the cylinder in a local $(\mathbf{x}, \mathbf{y}, \mathbf{z})$-base. Also, each cylindrical surface is identified by a random mesh.

\subsubsection{Rigid modes}

Generally, the modes defined by translation or rotation are valid for any surfaces. However, due to the degrees of invariance, all rigid modes are not relevant to determine the same for a cylindrical surface. Modes generated by translation and rotation along $\mathbf{z}$-axes are not significant and the surface remains unchangeable by these displacements. A rigid mode is characterized by a modal vector field and can be written according to the translation along $\mathbf{x}$-axis as given in Eq. (5):

$\mathbf{u}_{r-T x}(x, y, z)=\left(\begin{array}{l}1 \\ 0 \\ 0\end{array}\right)$.

The mode is defined by an amplitude coefficient equal to $+1 \mathrm{~mm}$ (see Fig. 2a). Generated rigid modes in the present work have the same amplitude values.

When considering a rotation along the $\mathbf{x}$-axis, a rigid mode can also be determined based on the SDT concept as given by Eq. (6):

$\mathbf{u}_{r-R x}(x, y, z)=\left(\begin{array}{c}0 \\ -z \cdot r_{x} \\ y \cdot r_{x}\end{array}\right)$

where $r_{x}$ denotes the amplitude of rotation (see Fig. 2b).

One mode $\mathbf{u}_{r-D}$ by expansion of the cylinder can be defined by variations of the radius. This mode is expressed by Eq. (7):

$\mathbf{u}_{r-D}(x, y, z)=\alpha \cdot\left(\begin{array}{c}\cos \left(\arctan \left(\frac{y}{x}\right)\right) \\ \sin \left(\arctan \left(\frac{y}{x}\right)\right) \\ 0\end{array}\right)$

where $\alpha$ is an expansion coefficient (see Fig. 2c).

In the following figures, nominal cylinder is illustrated in red and the rigid modes are shown by white color.

\subsubsection{Rippled modes}

The modal field $\mathbf{u}_{o}$ causes a translation of the surface with a sinusoidal amplitude with $z$ - parameter perpendicular to revolution axis, $\mathbf{z}$. In the following section, three examples of such class of modes are proposed (see Fig. 3):

For a sinus presentation within $[0,2 \pi]$, the modal field can be globally defined by:

$\mathbf{u}_{o}(x, y, z)=\left(\begin{array}{c}\sin \left(\frac{2 \pi}{\lambda} z\right) \\ \sin \left(\frac{2 \pi}{\lambda} z\right) \\ 0\end{array}\right)$

where $\lambda$ is the wavelength of the defect which can be related to the manufacturing process or to geometry itself if $\lambda=L$ ( $L$ is the length of the cylinder). Moreover, in this class of modes, banana modes can be identified compared to those generated by Henke et al. [28].

\subsubsection{Modes of section}

The modes of section are defined by one, a variation of the radius according to the coordinate $z$ on the axis of revolution and two, the angular position of a considered point. For the cylinder, some elementary modes of section can be identified such as taper, elliptic, hourglass and barrel modes. These modes represent generally the most and significant form defects of manufactured parts. However, the method can be generalized and applied to determine technological modes having impact on surfaces sections.

The taper mode is defined by amplitude of deviation which is proportional to the $z$-coordinate. Modal vector field associated to this mode is thus considered the same as the modal vector field for dilation with $\alpha=\beta . z$ in Eq. (6) where $\beta$ is the parameter to define taper geometry. In the case in this paper, the taper mode $\mathbf{u}_{s-T a p e r}$ with $\beta=\frac{\sqrt{2}}{L}$ ( $L=$ length of cylinder) is defined by Eq. (9):

$\mathbf{u}_{s-\text { Taper }}(x, y, z)=\frac{\sqrt{2}}{L} z \cdot\left(\begin{array}{c}\cos \left(\arctan \left(\frac{y}{x}\right)\right) \\ \sin \left(\arctan \left(\frac{y}{x}\right)\right) \\ 0\end{array}\right)$.

With the elliptic mode, the section of the cylinder is modified in a constant way over the entire length and takes the form of an ellipse instead of a circle. The definition of this mode is based on parametric representation of an ellipse in a Cartesian frame. The modal vector field $\mathbf{u}_{s-\text { Elliptic }}$ can thus be defined by Eq. (10):

$\mathbf{u}_{s-\text { Elliptic }}(x, y, z)=\left(\begin{array}{c}(r+1) \cos \left(\arctan \left(\frac{y}{x}\right)+\theta\right)-x \\ (r-1) \sin \left(\arctan \left(\frac{y}{x}\right)+\theta\right)-y \\ 0\end{array}\right)$

where $\theta$ is the angle between $(r+1)$-axis and $\mathbf{x}$-axis of the Cartesian frame.

The barrel mode corresponds to a quadratic variation of the radius along $z$-coordinate. The mode characterizes a parabolic profile of a part which can point out like a "barrel". It can be defined according to Eq. (6) with $\alpha=k z(z-L)$, where $k$ is a reel. Such mode for the cylinder can be expressed with Eq. (11):

$\mathbf{u}_{s-\text { Barrel }}(x, y, z)=\frac{4}{L^{2}} z(z-L) \cdot\left(\begin{array}{c}\cos \left(\arctan \left(\frac{y}{x}\right)\right) \\ \sin \left(\arctan \left(\frac{y}{x}\right)\right) \\ 0\end{array}\right)$.

Fig. 4 shows three examples of the modes considered in this section.

In addition to these modes, other modes often cited for cylindrical shapes can also be represented by MMD, like the hourglass, lobing and random modes as illustrated in Fig. 5.

Furthermore, in this article, to characterize and generate classical form defects when dimensioning parts, the different modal vector fields defined below are normalized to ensure that amplitudes are metric coefficients. It is therefore, proposed to apply the maximum norm to modal vector fields in all mesh nodes of the cylinder and to make it equal to $+1 \mathrm{~mm}$ according to the relation in Eq. (12):

$\max \left(\left\|\mathbf{u}_{i}(\mathrm{M})\right\|\right)_{1 \leq i \leq n_{\text {modes }}}=1[\mathrm{~mm}], \quad \forall \mathrm{M}$ in the cylinder, $n_{\text {modes }}$ denotes the number of modes. 

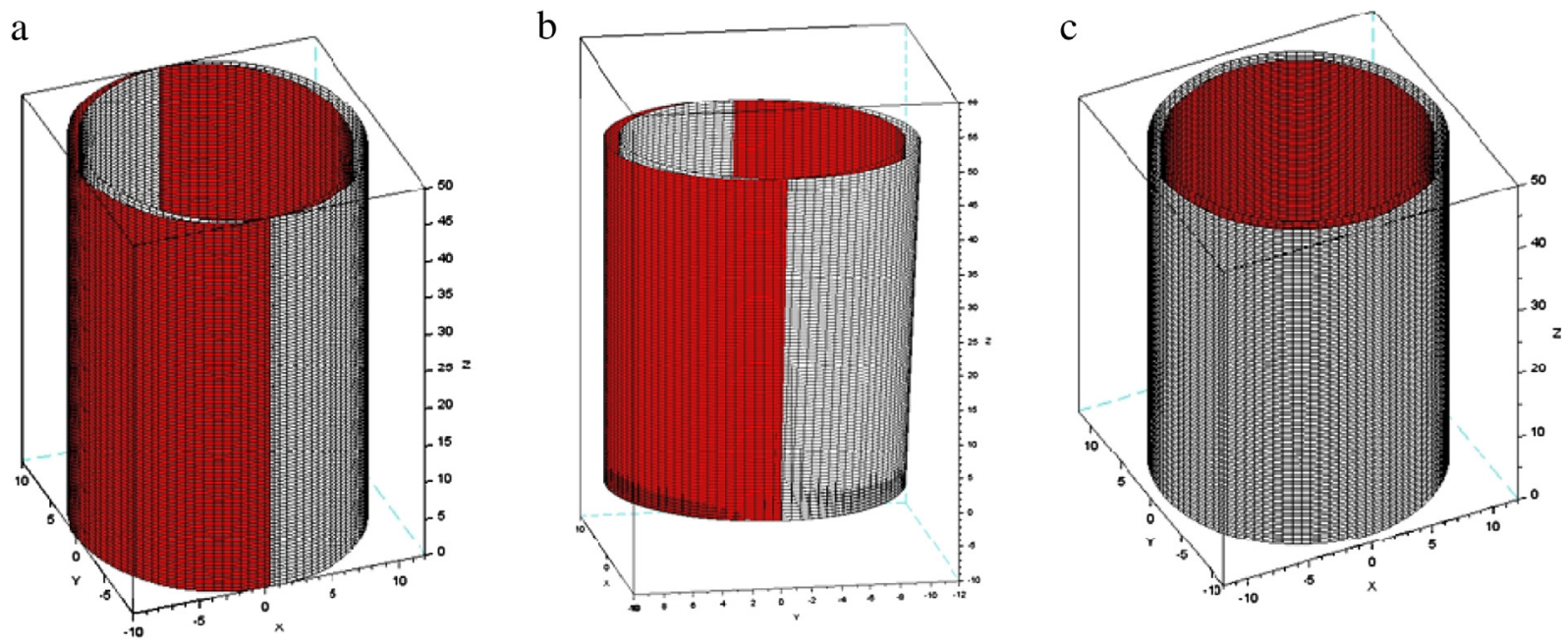

Fig. 2. Rigid modes of the cylinder: (a) translation (b) rotation and (c) expansion.

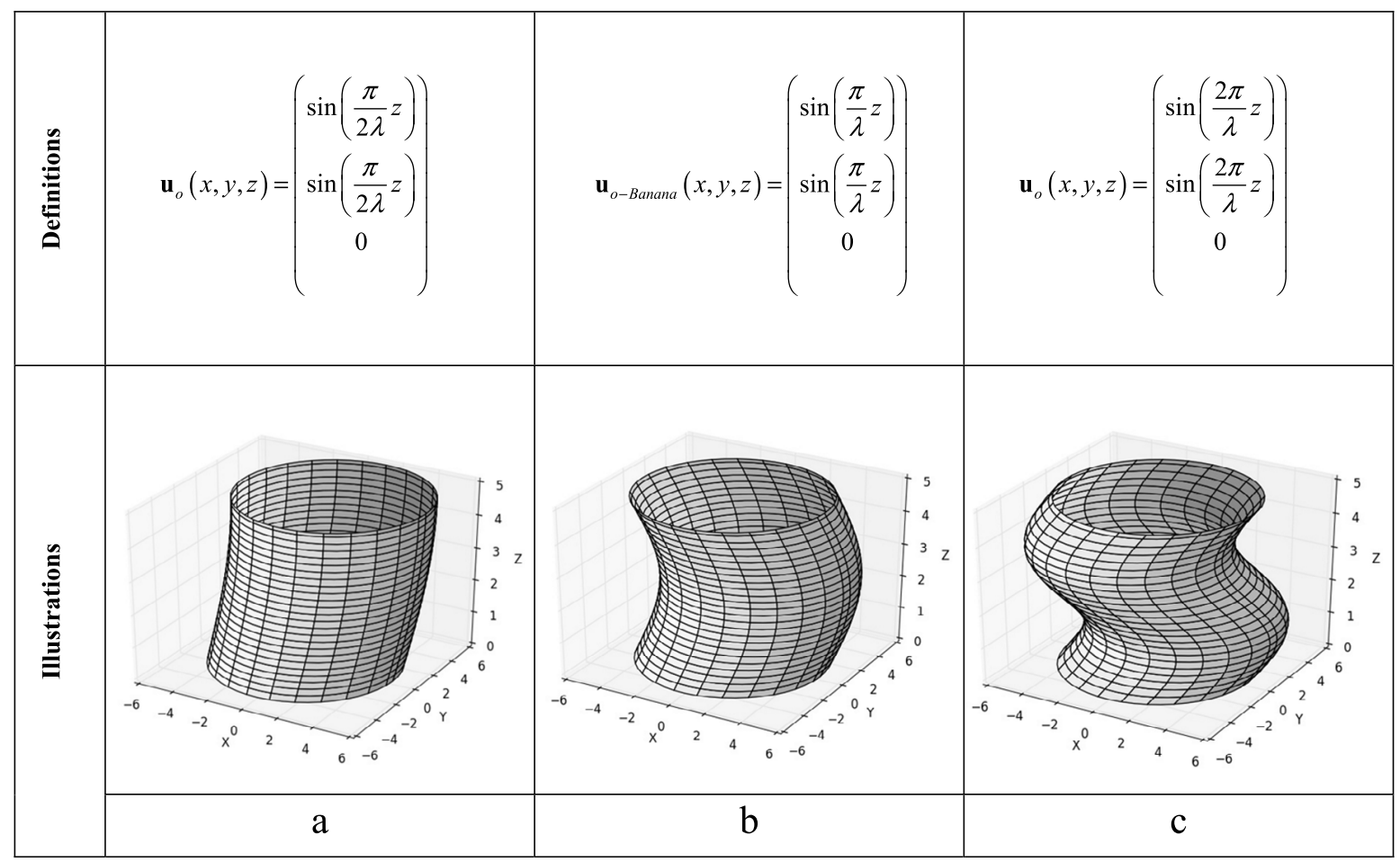

Fig. 3. Examples of rippled modes (banana, ... modes).
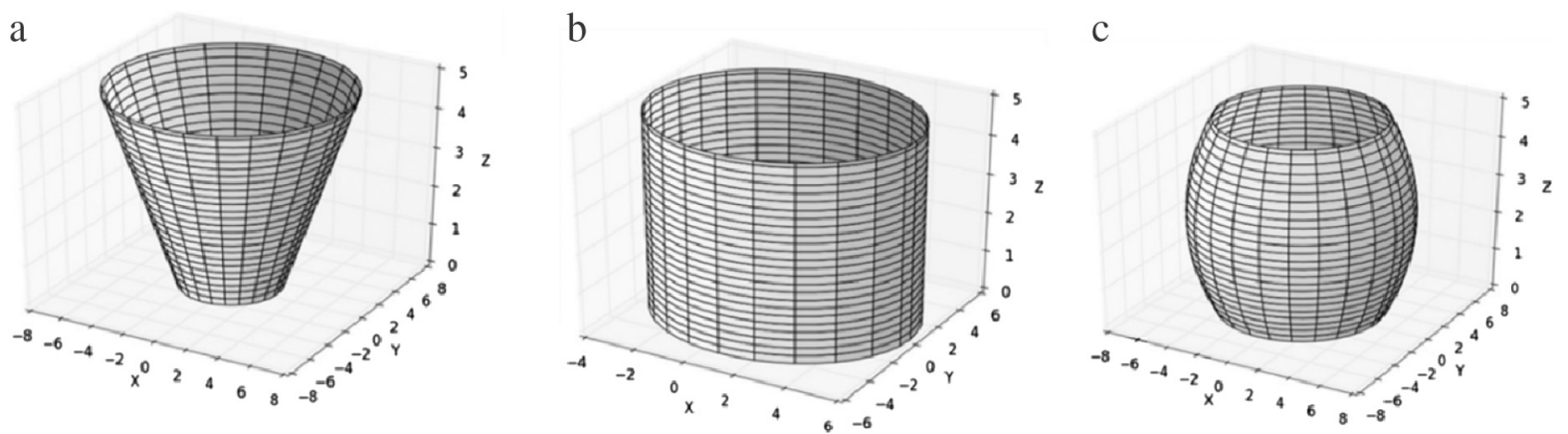

Fig. 4. Taper, (b) elliptic and (c) barrel modes. 
a

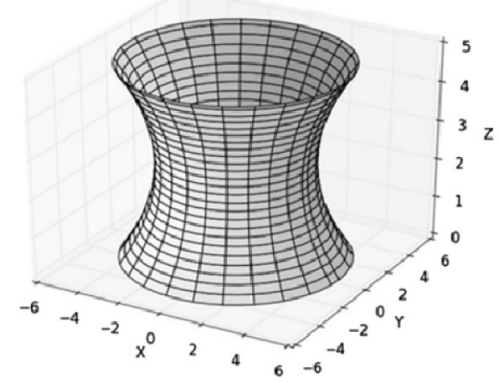

b

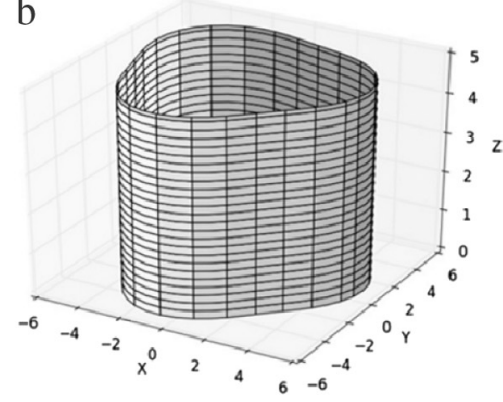

C

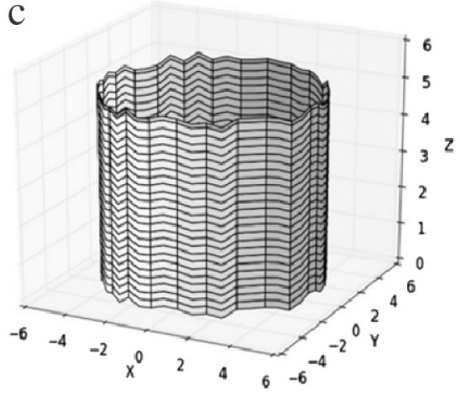

Fig. 5. (a) Hourglass (b) lobing and (c) random modes.

Finally, the discussed modes can thus be defined and normalized to build a modal basis.

All metric modes $\mathbf{u}_{i}$ represented below and their amplitude coefficients $\lambda_{i}$ define the MMD characterizing cylinder's form defects. Moreover, based on the MMD application, a non-ideal surface can be defined by the vector field as depicted in Eq. (13):

$\mathbf{u}=\sum_{i} \lambda_{r i} \mathbf{u}_{r i}+\sum_{i} \lambda_{o i} \mathbf{u}_{o i}+\sum_{i} \lambda_{s i} \mathbf{u}_{s i}, \lambda_{r i}, \lambda_{o i}, \lambda_{s i} \in \mathbb{R}$.

In order to optimize the computing time to generate form defects by implementing the MMD and the sum (refer to Eq. (13)), the modal representations are defined through matrix forms. The modal vector field $\mathbf{u}$ is henceforth represented by a matrix equal to the addition of the elementary modal matrices associated to $\mathbf{u}_{r i}$, $\mathbf{u}_{o i}$ and $\mathbf{u}_{s i}$.

Fig. 6 illustrates the definition of a non-ideal cylinder according to the MMD application as per Eq. (13).

\subsubsection{Overall approach for form defects modeling}

The MMD, one of the continuous methods, is proposed in this paper to generate cylinder's form defects. The approach is developed for a required parametrization of surfaces to deal with defect probability computation in an assembly simulation procedure. The assembly simulation is moreover, currently based on constrained optimization problems and defect probability assessment $[4,47,58]$. To add value to the existing research, the proposed MMD integrates form defects into simulation. In addition, the constraints of contacts between parts' surfaces nominally in contact include more new parameters which are identified by modes of form defects in the constrained optimization problem.

The novelty of the method can be recognized by its implementation on two fronts. One, on the definition of modal vector fields derived from the geometric characteristics of the surfaces, and two, on the application of simple algebraic relations. Non-ideal surface is hence defined as superposition of the nominal surface and the defects are modeled by vector field of displacements. For any point, $\mathrm{M}$, of a nominal surface, the point $\mathrm{M}^{\prime}$ belonging to the non-ideal surface is defined by: $\mathrm{M}^{\prime}=\mathrm{M}+\mathbf{u}(\mathrm{M})$ and the modal vector fields are generally normalized to build a modal basis. The first part of the subject paper deals with the vector modal determination as well. The modes, center of the MMD method, are defined form the manufacturing process. They are defined by a designer based on his/her manufacturing knowledge. Further, when measurement data are defined, modes can be deduced by fitting operation by least square method. Currently, the method is applied to the cylinder and classical modes are determined, implemented to the plane, and can be extended to complex geometries.

\section{Assembly simulation taking parts' form defects into account}

The following section of the paper will attempt to handle assembly simulation of non-ideal parts by mathematical and conceptual basis for the proposed method designated by MMD (see Fig. 7).
Cylinders, which are potentially in contact, are designated by the numbers 1 and 2 complying with the assumption as follows:

Assumption 1. External surfaces in the cylindrical pair are assigned with an odd (1) index while inner surfaces are assigned with an even index (2).

\subsection{Consideration of form defects in cylindrical pairs}

The cylinders are discretized into a mesh of points using Shannon's sampling theorem [59]. Corresponding points are then considered in the cylinders meshes. Since, nominal cylinders have different lengths, the longest cylinder is truncated and re-meshed in order to consider only functional surface in joint. The employed method to analyze the cylindrical pair can be described by the following steps: (1) comparing the lengths of the cylinders, (2) remeshing of cylinder $C_{2}$ using the same length $L_{1}$ of $C_{1}$, (3) application of the MMD method on the cylinders, and (4) application of all modes of $C_{2}$ to the resulting one by truncation.

Since, MMD is particularly used to characterize form defects of cylinders, for the case of assembly simulation, difference surface (or deviation surface) $[23,30]$ was employed. The relative positioning problem between surfaces that do not have perfect geometric form and are potentially in contact, is converted to the relative positioning problem between the difference surface and one of the perfect surfaces. The built difference surface takes into consideration all stacked form defects of surfaces in contact and was computed initially based on the modal decomposition employing the FEA $[30,60]$. The assembly analysis resulted from the computation of the clearance torsor (SDT) to characterize gaps between surfaces in contact. Globally, a torsor represents all relative displacements allowing the contact between surfaces, is defined by six components (three translations denoted by $u, v$ and $w$ and three rotations designated by $\alpha, \beta$ and $\gamma$ ), and depends on the surface class (plane, cylindrical, or spherical) to be modeled. It can be written in any point $\mathrm{M}$ as per Eq. (14):

$\{\mathbf{T}\}_{\mathrm{M}}=\left\{\begin{array}{ll}\alpha & u \\ \beta & v \\ \gamma & w\end{array}\right\}_{\mathrm{M}}$.

The relative positioning issue of parts with form defects based on optimization problem was treated in few works in the literature. Pierce and Rosen $[8,61]$ proposed a mathematical programming formulation for the tolerance analysis of end-milled steel and aluminum components. The NURBS formulation was employed to represent features that must be modeled using higher-order polynomials through the proper selection of control vertices, knot and weight values that can in turn represent localized features. However, the implemented algorithm should minimize the distances between any point on one feature and the corresponding closest point of the other feature to avoid interference of parts. 

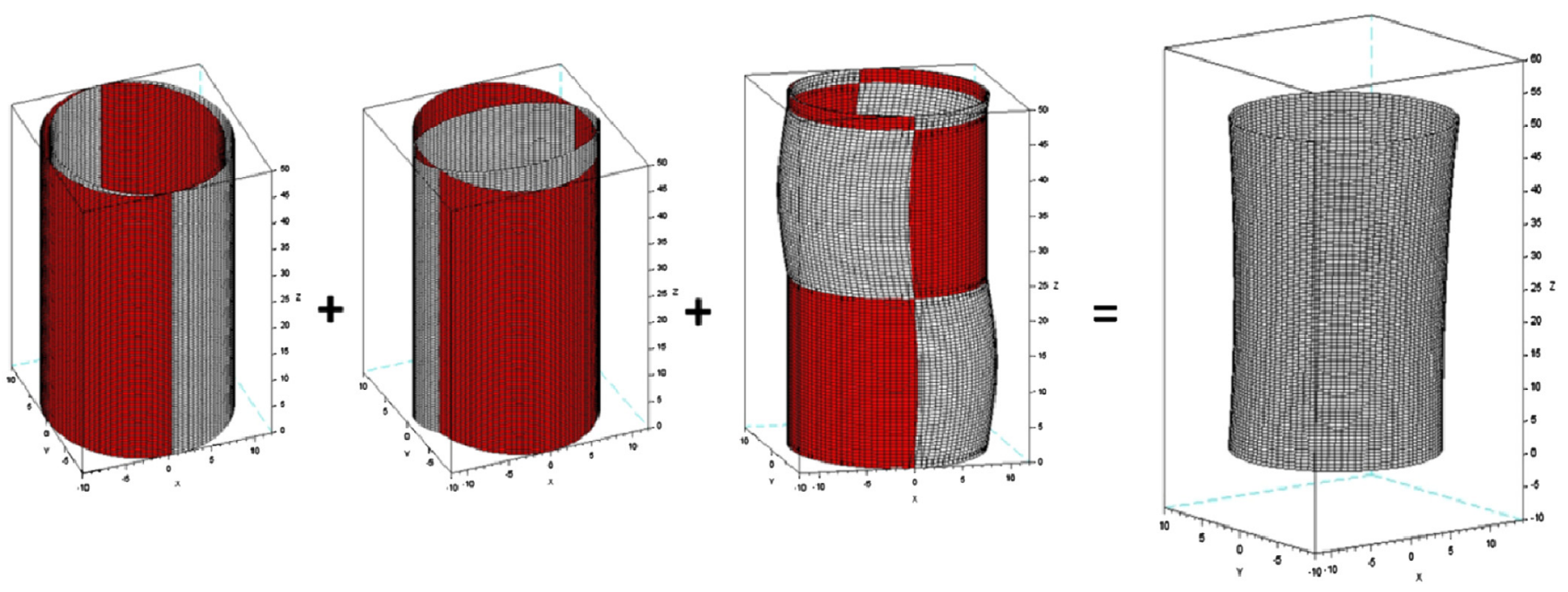

Fig. 6. Example of generation cylinder form defects based on MMD.

a

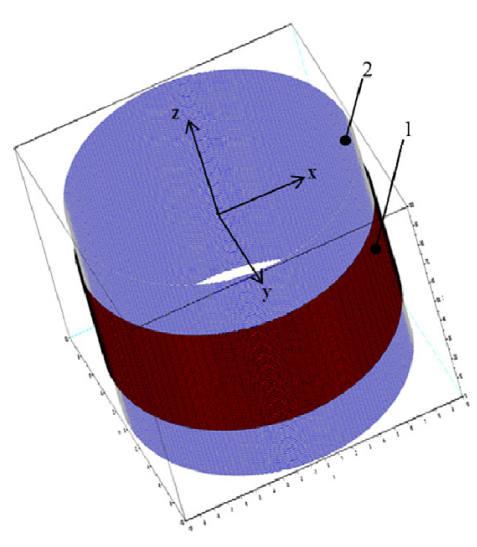

b

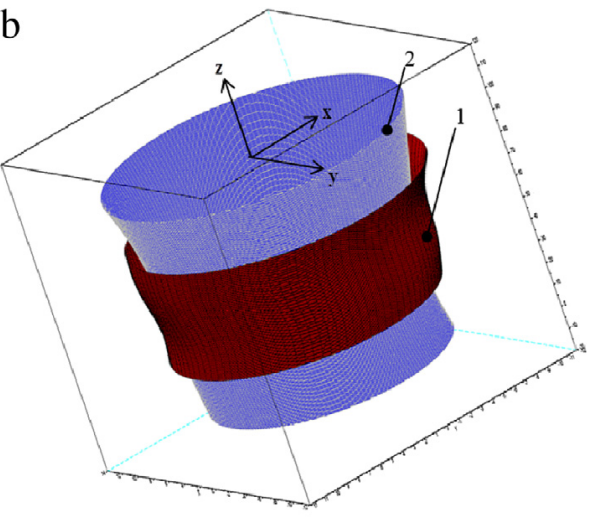

Fig. 7. (a) Nominal surfaces and (b) non-ideal cylinders potentially in contact.

According to Schleich et al. [11,23], the relative positioning of nonideal parts is defined as the relative positioning of the two associated Skin Model Shapes. The developed approach was based on the registration of the point clouds. The pairwise registration was then generated by the Iterative Closest Point (ICP) algorithm. The algorithm iteratively revised the transformation (combination of translation and rotation) needed to minimize the distance from the source to the reference point cloud [62]. The proposed approach for optimization is based on the concept of difference surface and the introduction of signed distance.

\subsection{Difference surface-based method}

The concept of difference surface gives the opportunity to consider superimposed form defects of surfaces which are potentially in contact. Generated by the MMD, form defects are particularly deferred on only one of the nominal surfaces. The joint is then resulted between a perfect surface and a non-perfect surface. The associated modes of the difference surface are by Eq. (15):

$\lambda_{d f, i} Q_{d f, i}=\lambda_{2, i} Q_{2, i}-\lambda_{1, i} Q_{1, i}, \forall 1 \leq i \leq n$

where $Q_{d f, i}$ is the $i$ th mode of the of difference surface, and $Q_{1, i} \&$ $Q_{2, i}$ are the modes of surfaces $1 \& 2$, respectively.

The difference surface is employed in tolerance analysis procedure to simplify computing of the distance between surfaces and subsequently, to minimize computing time. Indeed, the clearance torsor requires assessment of the distance between any two points of surfaces in a joint. Using the difference surface concept, the distance is computed from a perfect reference to the generated difference surface. In literature, the "real model" was considered to evaluate the distance between surfaces potentially in contact $[9,63]$. The equivalence between models has also been particularly assessed for 2D planar joints [63].

For the cylindrical pairs, the difference surface is defined according to MMD method. If a cylinder 1 is considered as a perfect cylinder and a cylinder 2 is defined by the superposition of all $\mathbf{u}_{1, i}$ and $\mathbf{u}_{2, i}$ of surfaces 1 and 2 , respectively, then each point $\left(x_{d f}, y_{d f}, z_{d f}\right)^{T}$ in the difference surface can be defined in a $(\mathbf{x}, \mathbf{y}, \mathbf{z})$ base according to Eq. (14) as follows in Eq. (16):

$$
\left(\begin{array}{l}
x_{d f} \\
y_{d f} \\
z_{d f}
\end{array}\right)=\left(\begin{array}{l}
x_{2} \\
y_{2} \\
z_{2}
\end{array}\right)+\sum_{i=1}^{n} \lambda_{1, i} \mathbf{u}_{i}\left(x_{1}, y_{1}, z_{1}\right)-\sum_{i=1}^{n} \lambda_{2, i} \mathbf{u}_{i}\left(x_{2}, y_{2}, z_{2}\right)
$$

where $\mathbf{u}_{i}$ is the modal vector field, and the points $\left(x_{1}, y_{1}, z_{1}\right)$ and $\left(x_{2}, y_{2}, z_{2}\right)$ are in cylinders $C_{1}$ and $C_{2}$, respectively. Moreover, each $\mathbf{u}_{i}$ is replaced by its matrix definitions $Q_{i}$ according to $\mathbf{x}, \mathbf{y}$ and $\mathbf{z}$ projections.

Fig. 8 shows two models of surfaces which are equivalent . If $\mathrm{N}_{1} \in C_{1}, \mathrm{~N}_{2} \in C_{2}$ and the correspondent points $\mathrm{M}_{1}$ and $\mathrm{M}_{2}$ in the non-ideal surface are modeled by MMD application, then the point, $\mathrm{M}_{d f}$ is defined by Eq. (16) in the difference surface, and 


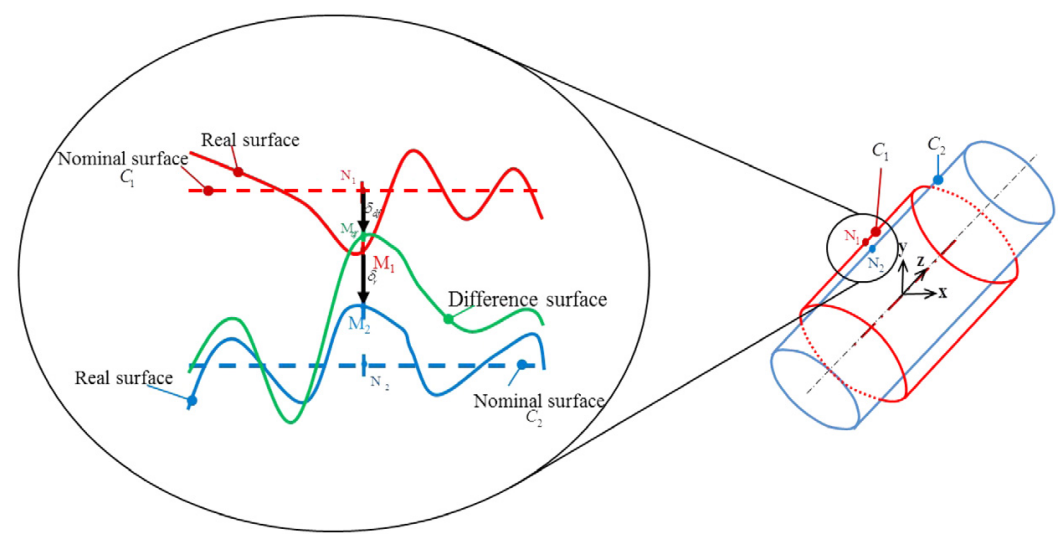

Fig. 8. Difference surface, real surfaces and designated distances: 2D projection.

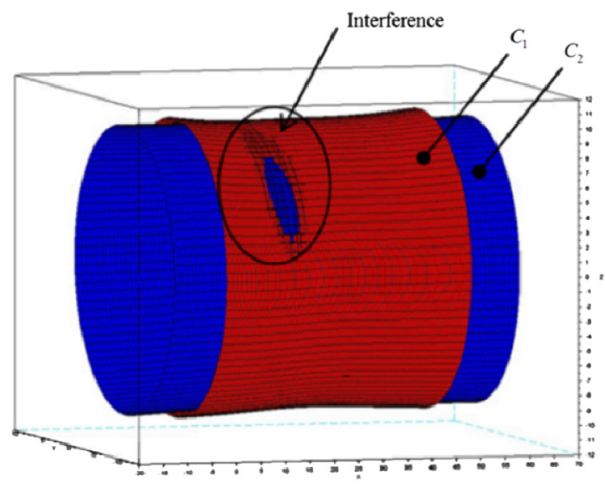

Fig. 9. Cylindrical pair with interference apparition.

$\delta_{d f}=\left\|\mathbf{N}_{1} \mathbf{M}_{d f}\right\|$ and $\delta_{r}=\left\|\mathbf{M}_{\mathbf{1}} \mathbf{M}_{\mathbf{2}}\right\|$ are the distances between surfaces considering the two models.

Also, if the modal vector fields $\mathbf{u}_{1}$ and $\mathbf{u}_{2}$ are computed in the points $\mathrm{N}_{1}$ and $\mathrm{N}_{2}$, then $\mathbf{u}_{1}=\mathbf{N}_{\mathbf{1}} \mathbf{M}_{1}, \mathbf{u}_{2}=\mathbf{N}_{2} \mathbf{M}_{2}$ and the expression $\mathbf{u}_{2}-\mathbf{u}_{1}=\mathbf{N}_{2} \mathbf{M}_{d f}$ is obtained.

Finally, the expression below is generated:

$$
\begin{aligned}
\delta_{r} & =\left\|\mathbf{M}_{\mathbf{1}} \mathbf{M}_{\mathbf{2}}\right\|=\left\|\mathbf{M}_{1} \mathbf{N}_{1}+\mathbf{N}_{\mathbf{1}} \mathbf{N}_{\mathbf{2}}+\mathbf{N}_{\mathbf{2}} \mathbf{M}_{\mathbf{2}}\right\| \\
& =\left\|\mathbf{N}_{\mathbf{1}} \mathbf{N}_{\mathbf{2}}+\mathbf{u}_{2}-\mathbf{u}_{1}\right\|=\left\|\mathbf{N}_{\mathbf{1}} \mathbf{N}_{\mathbf{2}}+\mathbf{N}_{2} \mathbf{M}_{d f}\right\|=\delta_{d f} .
\end{aligned}
$$

The equivalence between model surfaces is then assessed. The clearance torsor which is also a fundamental key for assembly analysis, can further be evaluated and optimization results can be thus be addressed.

\subsubsection{Introduction of a simplified signed distance}

In contrast to other definitions of distances, the signed distance function is employed in this paper to identify and avoid interpenetrations between surfaces in contact (see Fig. 9). Interferences can appear in the initial configuration but do not imply directly the non-assembly, and an analysis should nevertheless be carried out to be sure. The signed distance can be seen as an indicator whether or not the parts will collide [11]. For perfect meshes, signed distance is the absolute value of Euclidian distance.

Cylinders $C_{1}$ and $C_{2}$ are non-ideals. If $\mathbf{n}$ is the outward pointing vector normal to the surface $C_{1}$ in any point $A$, then the signed distance $(d)$ is defined by the scalar product as shown in Eq. (17):

$d=\mathbf{A B} . \mathbf{n}, \mathrm{A} \in C_{1}$ and $\mathrm{B} \in C_{2}$.

The assessment of the signed distance between all corresponding pairs of points of the two surfaces appears costly in terms of running time and algorithmic complexity, compared to the Euclidian distance. It is also very important to propose a new simplified definition of the subject distance. Signed distance will then be assessed between points of the difference surface and the ideal reference and not between two points in the non-ideal surfaces. A simplified signed distance can be here used for the cylindrical pair joint according to a (x, y, z)-base as shown in Eq. (18):

$d=R_{1}-\sqrt{x_{\mathrm{B}}^{2}+y_{\mathrm{B}}^{2}}$

where $R_{1}$ is the radius of the nominal cylinder $C_{1}$ and $\mathrm{B}\left(x_{\mathrm{B}}, y_{\mathrm{B}}, z_{\mathrm{B}}\right) \in$ $C_{d f} . C_{d f}$ is the difference surface.

Simplified signed distance (see Eq. (18)) is specific to the studied geometry and must be appropriated for any other type of contact in a local base. Since the definition of the signed distance is performed in relation with the difference surface-based method, the components of clearance torsor must be determined to let the method be suitable for tolerance analysis procedure.

\subsubsection{Assembly simulation and optimization}

The assembly requirement was given by Qureshi et al. [4]: "For all admissible deviations, there exists a gap configuration such as the assembly requirements and the behavior constraints are respected". The technique to compute the requirement verification is to minimize one constraint with respect to all geometrical constraints. A solution would mean that the assembly is possible, and vice versa.

To analyze the effects of form defects on assembly simulation, it is necessary to define the optimization problem and to select the adequate algorithm that returns clearance torsor components. The aim of this section is to deal with the related optimization problem and to review some existing algorithms. If $\mathrm{N}(x, y, z)$ is a point in a chosen ideal surface and $\mathrm{M}\left(x_{d f}, y_{d f}, z_{d f}\right)$ is the correspondent point in the difference surface, then the adjusted position between surfaces is obtained by minimizing an objective function (function of the gap between surfaces) subject to a function of the torsor components with the signed distance between the two points $\mathrm{N}$ and $\mathrm{M}$ as the constraint.

The clearance torsor associated to the cylindrical pairs is defined in Eq. (19), where 'O' is the joint origin:

$\{\mathbf{T}\}_{0}=\left\{\begin{array}{ll}\alpha & u \\ \beta & v \\ 0 & 0\end{array}\right\}_{0}$.

Based on the definition of the torsor, the point $\mathrm{M}$ of the difference surface can be expressed as Eq. (15) and the objective function can be thus be written as Eq. (20):

$\min _{\alpha, \beta, u, v} f(\alpha, \beta, u, v)$. 
As the optimization problem in this paper has a constraint defined as the signed distance between surfaces in points $\mathrm{M}$ and $\mathrm{N}$, the equation for the constraint can be written as shown in Eq. (21):

$\operatorname{Dis}(\mathrm{N}, \mathrm{M}):=\sum \mathbf{N M} \cdot \mathbf{n}=\sum\left(\mathrm{N}_{x, y, z}-\mathrm{M}_{x, y, z}\right) . \mathbf{n}$

where $\mathbf{n}$ is the normal vector of the ideal surface at the point $\mathbf{N}$.

Generally, the optimization problem can globally be defined by Eq. (22):

$$
\min f(\alpha, \beta, u, v)
$$

$\operatorname{subject~to} \operatorname{Dist}(\mathrm{N}, \mathrm{M}) \geq 0 \quad \forall \mathrm{N}, \forall \mathrm{M}$.

Due to the aim of the optimization problem, the objective function is defined as: $f(\mathbf{x})=\frac{-1}{2}\left(u^{2}+v^{2}+\alpha^{2}+\beta^{2}\right)$. In fact, the main goal is to find the values of the clearance torsor (gaps) such that the assembly constraints are satisfied. The definition of the objective function impacts only the computing time. Dantan [22] compares several optimization formulations and demonstrated that this considered function gives significant results in terms of time consumption.

The constraints associated to the optimization problem are defined below in Eq. (23):

$\mathbf{d}_{A_{i j}} \cdot \mathbf{n}_{i j} \leq D_{i j}$

where $\mathbf{d}_{A_{i j}}$ is the displacement in the point $A_{i j}$ of cylinder $2, \mathbf{n}_{i j}$ is the normal vector to the nominal surface, and $D_{i j}$ is the signed distance between $A_{i j}$ and the cylinder.

A solution would reveal the existence of an admissible configuration of gaps such that the assembly requirement is verified. If no solution is provided, then the assembly is not possible because there is at least one interference between two components of the assembly.

Several algorithms were applied and then compared based on two criteria: computing time, and accuracy of the assembly assessed by the signed distance between surfaces. The evolution of the computing time of algorithms was also taken into account. Among the various existing algorithms, HLRF algorithm (see Appendix), proposed by Hasofer and Lind [64] and Rackwitz and Fiessler [65], was employed. The algorithm requires least amount of storage, has lower number of computations and converges fast for most situations [66]. The HLRF algorithm was improved in this paper and combined with iHLRF algorithm that was developed by Zhang and Kiureghian [67] by introducing a non-differentiable merit function and an Armijo rule to select the step size in the linear search. The iHLRF algorithm was implemented with constraint defining the signed distance between cylinders $C_{1}$ and $C_{2}$. The algorithm converged in 8 iterations. Moreover, it was made evident that efficient solutions were controlled according to both the numerical value of the signed distance and the components of the SDT (see Fig. 10).

Further, some more algorithms were investigated such as $\mathrm{Ge}-$ netic Algorithm (GA) which was employed to minimize the signed distance by converging it to zero. The GA provided a less accurate result than the iHLRF algorithm and did not ensure precise results. For 2601 generated points in surfaces, the GA took 30 times more time than iHLRF for five different simulations using the same modes amplitudes. Linear Matrix Inequality (LMI) algorithm was found to make it possible to solve a linear problem based on some inequalities of matrix [68]. The algorithm required square matrix which was not the case of the optimization problem in this paper. Nelder-Mead algorithm [69] is also a direct search algorithm based on the simplex method [70].

Quapro (see Appendix) is a linear quadratic programming solver proposed in the ATOMS module. It uses the QR-decomposition method and the Chloesky factorization of the Hessian matrix of

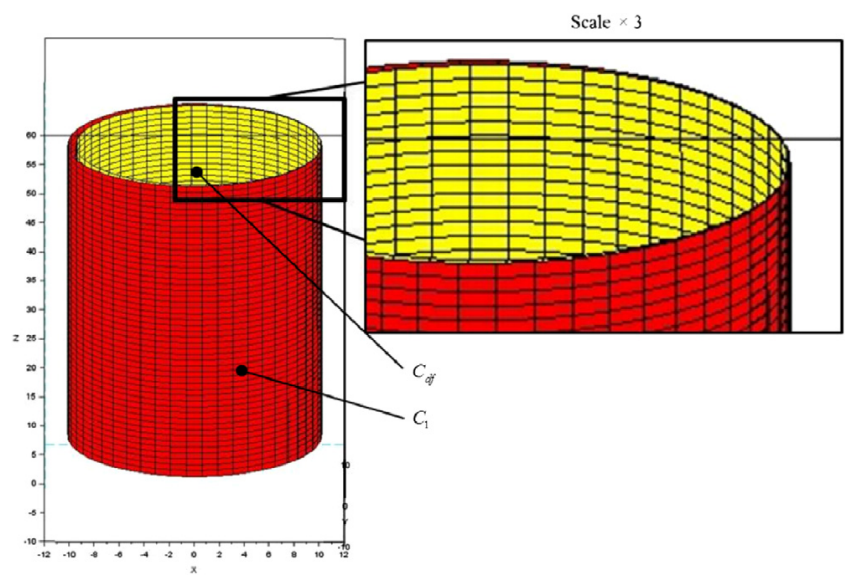

Fig. 10. Perfect cylinder $C_{1}$ and difference surface $C_{d f}$ - adjusted cylindrical pair.

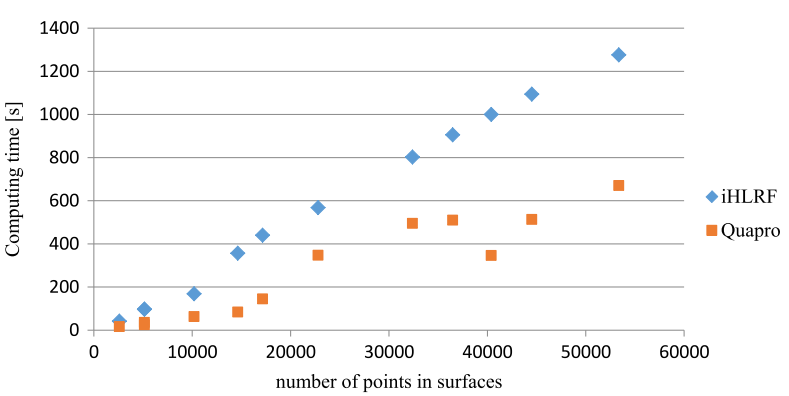

Fig. 11. Computing times of iHLRF and Quapro algorithms.

constraints for optimization [71]. The objective function for that optimization problem is defined by Eq. (24):

$$
\begin{gathered}
\qquad f(\mathbf{x})=\frac{1}{2} \mathbf{x}^{T} \mathbf{H} \mathbf{x}+\mathbf{p}^{T} \mathbf{x} \\
\quad \text { Ax } \leq \mathbf{b} \\
\text { Subject to }{ }_{c_{\text {inf }, i} \leq x_{i} \leq c_{\text {sup }, i}, \quad 1 \leq i \leq n}
\end{gathered}
$$

where $\mathbf{H}$ is an $n \times n$ symmetric matrix while $\mathbf{p}$ and $\mathbf{b}$ are $n$-vectors. In the case of the cylindrical pair, the input vector $\mathbf{x}$ is defined by non-zero components of the clearance torsor: $\mathbf{x}=$ $\left(\begin{array}{llll}u & v & \alpha & \beta\end{array}\right)^{T}$.

The objective function in this case is defined by $f(\mathbf{x})=$ $\frac{-1}{2}\left(u^{2}+v^{2}+\alpha^{2}+\beta^{2}\right)=\frac{-1}{2}\|\mathbf{x}\|^{2}$ with

$\mathbf{p}=\mathbf{0}$ and $\mathbf{H}=\left(\begin{array}{cccc}-1 & 0 & 0 & 0 \\ 0 & -1 & 0 & 0 \\ 0 & 0 & -1 & 0 \\ 0 & 0 & 0 & -1\end{array}\right)$

Quapro finds all adjusted displacements with a precision of around $10^{-4} \mathrm{~mm}$ which is also the fixed value for this simulation. The algorithm includes a stopping criterion and converges after 80 iterations. The Quapro programming does not depend on a gradient calculation as for the case of iHLRF. Quapro and iHLRF algorithms converge similarly and a comparison based on the evolution of the computing time per the number of points in surfaces is illustrated in the Fig. 11.

Fig. 11 shows that iHLRF has a linear computing time, however, it is difficult to predict the computing time of Quapro algorithm. The linearity can be explained by iHLRF-resolution being direct. The variations with the linearity can be due to the fact that a decrease in the number of points tends to "smooth" the form defects and therefore, some new local minimums in the distance 
Table 1

Assembly's probability based on iHLRF and Quapro programming.

\begin{tabular}{llll}
\hline & iHLRF & Quapro & $\boldsymbol{\Delta P}$ \\
\hline Case 1 (20H7h7) & $56.4 \%$ & $57.6 \%$ & $1.2 \%$ \\
Case 2 (20H7g6) & $51.6 \%$ & $54.0 \%$ & $2.4 \%$
\end{tabular}

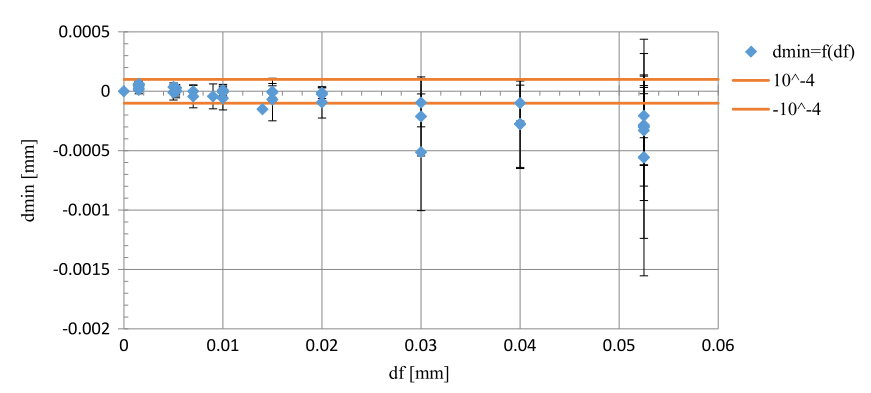

Fig. 12. Representation of $d_{\min }=f(\mathbf{d f})$.

can appear when the mesh is dense. Overall, Quapro algorithm is faster and more efficient than iHLRF despite being more irregular in performance and requiring more iterations to converge. Hence, it is more advantageous for use in optimization.

The probability of assembly is also assessed based on the algorithms. A Monte Carlo simulation with 1000 iterations on the same sets of points was randomly generated. The Table 1 illustrates a comparison between the algorithms when considering two ISO clearance fits (hole and shaft) [72].

Quapro algorithm converges faster than iHLRF without being significant ( $\Delta P<3 \%$ ). Quapro algorithm also includes a management of degenerated points which induce additional translations to let the surfaces have a suitable configuration for optimization.

In the following text, some numerical results illustrating the correlation between the signed distance designated by $d_{\min }$ and the generated form defect of the difference surface (see Fig. 12) have been shared. For small form defects, $d_{\text {min }}$ is lightly positive in the beginning and then becomes increasingly negative. If the form defect exceeds $0.00525 \mathrm{~mm}, d_{\min }$ can takes values below $-10^{-4}$. The difference surface-based method can then be used with a maximum form defect equal to $+0.00525 \mathrm{~mm}$ if the nominal gap is less than or equal to $1 \mathrm{~mm}$ (value used for simulation in the whole article). This criterion may seem restrictive but includes current case of clearance fits (cases 1 and 2 considered in the application) [72].

\subsection{Synthesis}

Real model and difference surface-base method are employed in this paper to simulate assembly of parts with form defects. This assembly simulation is based on mathematical optimization introducing signed distance constraint to a defined objective function with regards to the torsor of displacements. Some results of the classical cylindrical pair show that the two surface models are equivalents when the maximum of form defect generated by the MMD is not more than $0.00525 \mathrm{~mm}$ and the gap value does not exceed $1 \mathrm{~mm}$. In order to extend the assembly simulation of nonideal parts, the methodology will be applied to a simplified industrial over-constrained system. The aim is to determine the adjusted assembly configuration (particularly the torso components) with respect to the assembly joints' constraints.

\section{Application on an over-constrained mechanism}

The application is based on a simplified industrial electrical connector made up of two parts on which 4 cylindrical pair joints

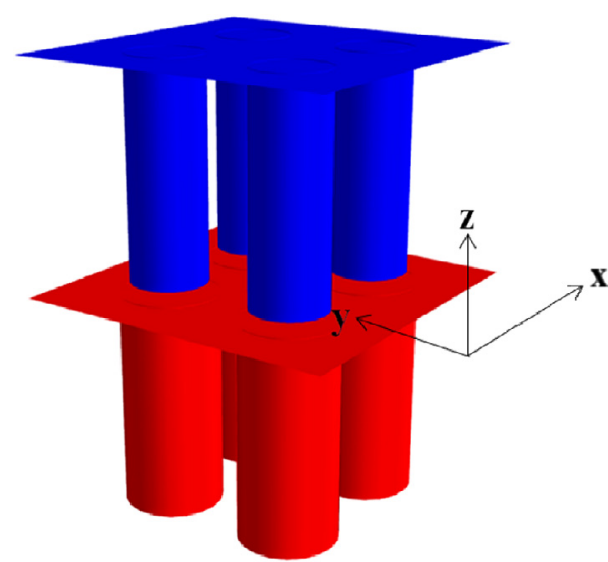

Fig. 13. Case study.

(4 pins must get into their compartments) are defined (see Fig. 13). By isolating functional surfaces, each surface can be characterized by a cylinder. The MMD developed in previous sections and the methodology for assembly simulation will be applied. The specifications of the two parts of the connector are detailed in the following figures. Fig. 14(a) illustrates the definition of the "hole" part and Fig. 14(b) represents the pin part definition.

In the case study, only functional surfaces are specified. To model the connector, geometric specifications are translated according to the MMD of the cylinder and the nominal radius of the hole and the pin are respectively equal to $0.9625 \mathrm{~mm}$ and $0.8075 \mathrm{~mm}$. The amplitudes for rigid modes are equals to $\lambda_{r}=$ $0.045 \mathrm{~mm}$, and for modes of section are equal to $\lambda_{s}=0.0125 \mathrm{~mm}$. The values are defined according to the associated position specification and the dimension of the tolerance zone of the diameters. Finally, the amplitudes of the rippled modes are respectively defined as $\lambda_{s p}=0.0125 \mathrm{~mm}$ and $\lambda_{s h}=0.01 \mathrm{~mm}$ for the pin and the hole parts, respectively.

Based on the MMD, the non-ideal connector can be represented in Fig. 15 where nominal connector is identified in the left of the figure.

The model used in the previous section to handle a cylindrical pair as an example was extended and thus adapted for any pinhole problem that can constitute generally a basic example of tolerance analysis of over-constrained assemblies. Several cylindrical pairs were considered together to ensure the assemblability of the connector and the assembly simulation was based on contained optimization. Considering an over-constrained assembly required firstly a global base on which the torsor components molding the contact were defined.

The assembly analysis aimed to modify the components of the clearance torsor. In the case study, the parallelism defined between the four cylindrical pair joints removed the degree of freedom associated to the rotation along $\mathbf{z}$-axis (which was bounded). Therefore, the clearance torsor, expressed in a global base can be defined by Eq. (25) [73]:

$\{\mathbf{T}\}_{0}=\left\{\begin{array}{ll}\alpha & u \\ \beta & v \\ \gamma & 0\end{array}\right\}_{0}$, where $\mathrm{O}$ is the origin of the base.

Since, individual joints in the mechanism cannot be considered independently, displacements according to the individual joints had an impact on the others. Also, as it becomes an ongoing challenge to evaluate all joints simultaneously, a unique functional surface including the four cylindrical surfaces was considered. The mathematical optimization previously performed from 
a

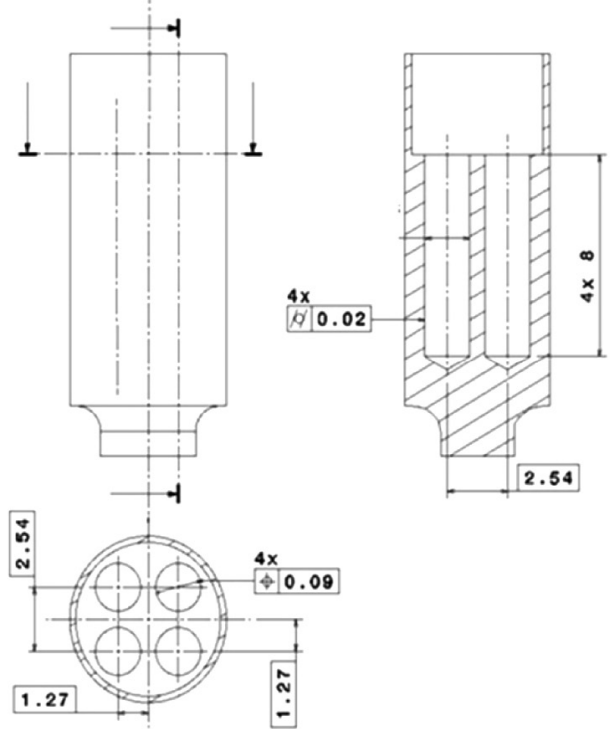

b

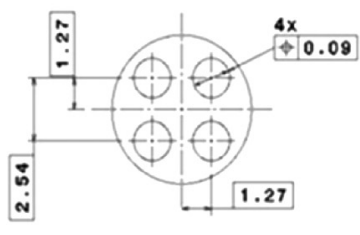

Section AA

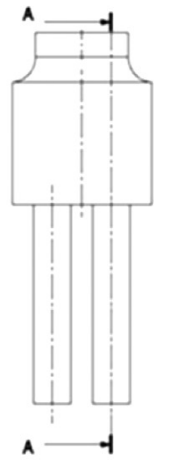

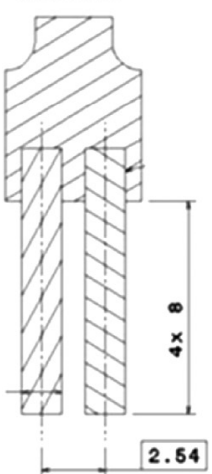

Fig. 14. Modified industrial connector. (a) Hole, (b) pin.
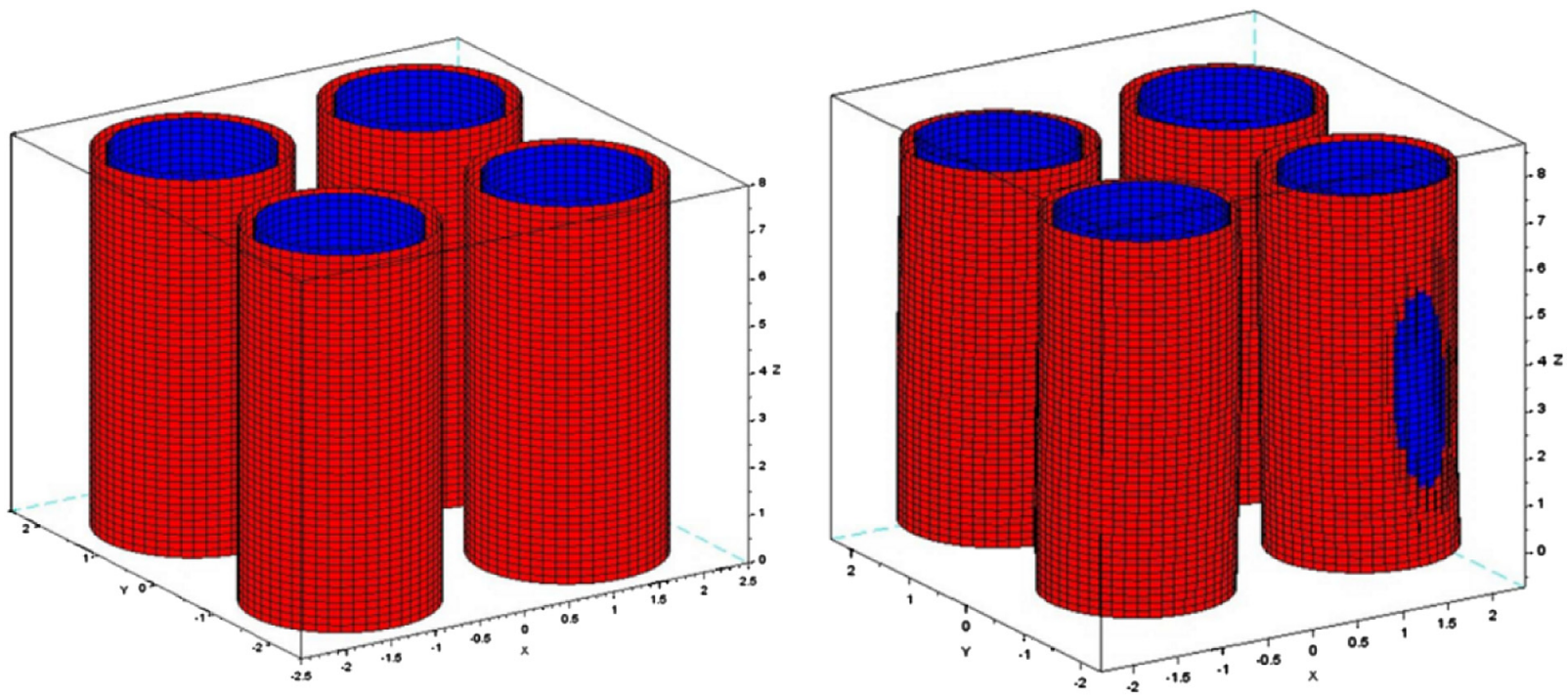

Fig. 15. Nominal and non-ideal parts geometries of the connector.

assigned distance between two surfaces and which subsequently allowed obtaining the adjusted configuration of contact, was performed considering the modified functional surface. The employed method may be summarized in the following way:

- Generation of real surfaces of the connector,

- Application of MMD to 4 cylindrical surfaces linear optimization with Quapro algorithm to determine the torsor components characterizing the adjusted configuration of contact position, and

- Evaluation of the distance between non-ideal parts.

The clearance torsor was held relevant if visually there were no interferences between surfaces in contact and if numerically the residual distance was maximum around $10^{-3} \mathrm{~mm}$. However, the difficulty consisted of precisely when the optimization did not converge for cases where a visual check ensured a solution. Indeed, for some contact configurations where the surfaces interfered in the initial position, Quapro algorithm did not give a favorable position with optimization using only the translation components.
The processing of the system thus ensured an addition of a rotation component according to the $\mathbf{z}$-axis. Therefore, one additional rotation component was introduced if degenerate points of the linear algorithm appeared. Such degenerated points of the interior surfaces (pins) had the capacity to return in the exterior surface. To ensure the efficiency of the given result of Quapro algorithm, it was compared with the iHLRF result as established for an individual cylindrical pair in the previous section.

The two algorithms were compared in 20 treatments of the case study where form defects were randomly generated according to the defined specifications in parts. Table 2 illustrates the computed distance by optimization and the computing time.

The results highlighted a second challenge when several joints in a mechanism were considered, i.e., the degradation of precision. Indeed, both algorithms included a stopping criterion as mentioned previously. The distance resulted from the optimization was however, higher. Although iHLRF was faster than Quapro algorithm, it had much lower accuracy. Quapro was then selected for optimization keeping in view its efficient results. The final adjusted assembly configuration is illustrated in the Fig. 16. 
Table 2

Accuracy and computing time comparisons.

\begin{tabular}{lll}
\hline & Quapro & iHLRF \\
\hline $\begin{array}{l}\text { Order of magnitude of the distance after optimization } \\
\text { Computing time }\end{array}$ & $\left(10^{-4}-10^{-3}\right) \mathrm{mm}$ & $\left(10^{-2}-10^{-1}\right) \mathrm{mm}$ \\
& $(30-150) \mathrm{s}$ & $(20-40) \mathrm{s}$ \\
\hline
\end{tabular}

Table 3

Assembly simulation taking into account all modes for form defects then only rigid modes.

\begin{tabular}{lll}
\hline & Form defects (including all modes) & Only rigid modes \\
\hline Successful assembly & 4912 & 4983 \\
Probability of assembly & $98.24 \%$ & $99.66 \%$ \\
\hline
\end{tabular}

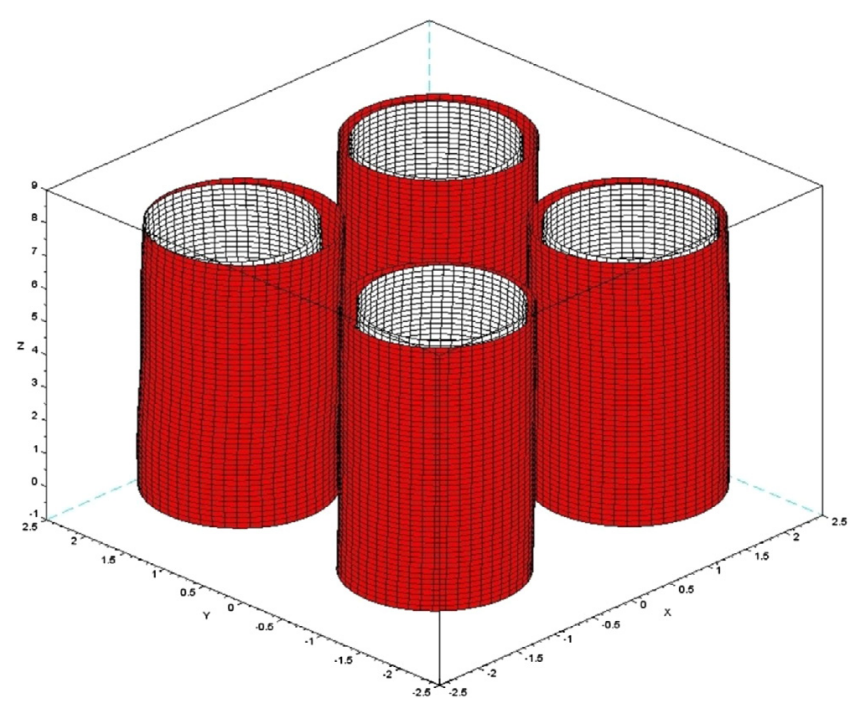

Fig. 16. Adjusted configuration of assembly of the connector.

The tolerance analysis approach was further based on Monte Carlo simulation with 5000 examples of connectors that considered cylindrical surfaces as non-ideals. The aim of the model was to estimate the probability of assembly with respect to some previous works [37]. Globally, the probability of assembly was equal to $98.24 \%$ and the associated assembly simulation probabilities are given in Table 3.

In this section, a common issue in tolerance analysis defined by cylindrical pairs was processed. The study case was a basic example of an over-constrained mechanism. Working on assembly simulation for such systems requires some adjustments when handling with optimization problem to determine the adjusted contact position between parts. The two major problems identified in simulation were related to one, the convergence and two, the degradation of the accuracy of employed optimization techniques. It was also extracted that the adjunction of a rotational component on the torsor could resolve the problem of identifying the contact position between two parts of the case study.

\section{Conclusion}

This paper dealt with developing and implementing a tolerance analysis approach considering parts' form defects. In the first part, the MMD method is proposed to generate form defects of cylinders. It is important to consider this class of surface when handling over-constrained systems. The methodology could be extended for complex surfaces and subsequent technological modes could be defined based on this method. The paper distinguished three types of modes generated by the MMD method globally for a cylinder: rigid modes, elliptic modes and banana modes.
In the second section, assembly simulation was introduced when a basic cylindrical pair was proposed. In the simulation, two surface models (i.e., real model and difference surface-based method) were implemented to determine the best fit defined by a torsor (rotations and translations) positioning of two nonideal cylinders. The torsor components were performed by linear and non-linear optimization problems considering two main algorithms; Quapro and iHLRF. Few criteria deduced to ensure the equivalence between the two surface models when assembly simulation occurred. Assembly simulation also required considering one optimization algorithm, Quapro and iHLRF. Therefore, these algorithms were compared in terms of computing time and accuracy of adjusted configuration of the contact.

An over-constrained mechanism which was a simplified industrial connector composed by two parts was analyzed further as a case study. It was deduced that more realistic results can be reached by modeling the whole contact among cylindrical features. For the torsor modeling, the adjusted configuration of contact was first determined by mathematical optimization and then by a Monte Carlo simulation to compute the probability of assembly of the two non-ideal parts.

The presented work overcame the issue of not considering the form defects in the assembly simulation. However, it focused on the integration of the form defects into tolerance analysis procedure to deal with compliant assemblies through parts' specifications. As part of future work, the research will be continued to characterize form defects of complex parts using the MMD method. The authors are already working on the extension of the methodology employed in this paper to more over-constrained assemblies in conjunction with ISO tolerance specifications. Finally, meshing Strategies of surfaces were studied to compare the results of the methodology. The issues of statistics on the modes amplitudes will also be addressed in future work.

\section{Appendix}

The HLRF algorithm originally developed by Hasofer and Lind [64] and later extended to non-normal random variables by Rackwitz and Fiessler [65], is perhaps the most popular algorithm used to solve the constrained optimization problem in structural reliability analysis. It is well known that the original form of the algorithm is unstable and may not converge under certain conditions.

$u^{*}=\arg \min \{\|u\|, G(u)=0\}$.

Zhang and Kiureghian [67] improved this algorithm by adding a line search scheme. The improved algorithm was denoted as iHLRF. The key steps are the same for all algorithms considered here and can be synthetized as follows (see Fig. 17):

- Choose a starting point $\mathbf{u}_{0}$, generally the origin of the space in the absence of specific information $(k=0)$,

- Evaluate the limit-state function $G\left(\mathbf{u}_{k}\right)$, 


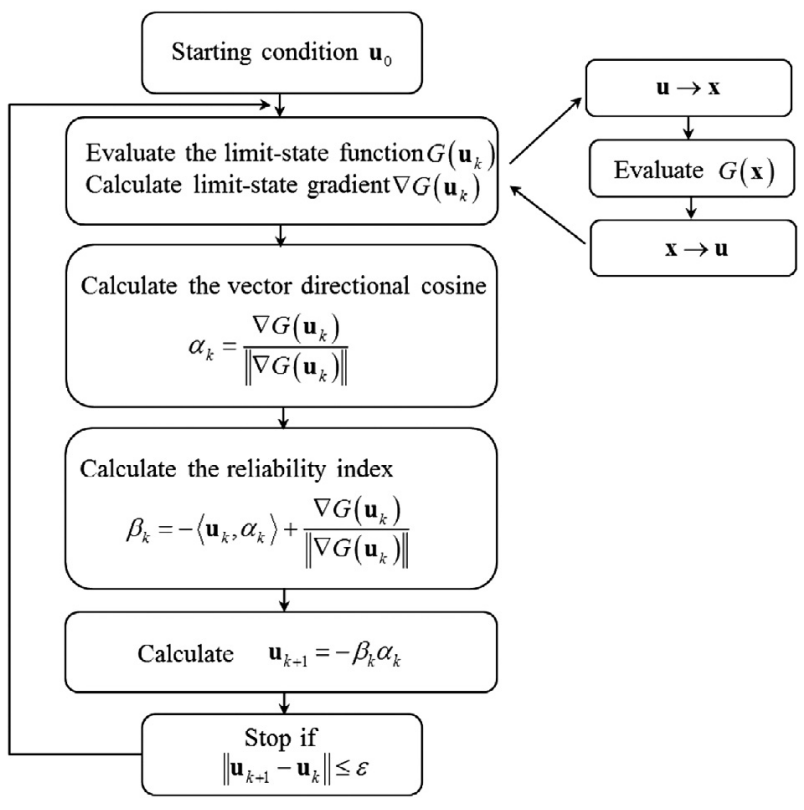

Fig. 17. The HLRF algorithm.

- Calculate the limit-state gradient $\nabla G\left(\mathbf{u}_{k}\right)$ and its norm $\left\|\nabla G\left(\mathbf{u}_{k}\right)\right\|$, and then deduce $\alpha_{k}=\frac{\nabla G\left(\mathbf{u}_{k}\right)}{\left\|\nabla G\left(\mathbf{u}_{k}\right)\right\|}$,

- Calculate $\beta_{k}: \beta_{k}=-\left\langle\mathbf{u}_{k}, \alpha_{k}\right\rangle+\frac{\nabla G\left(\mathbf{u}_{k}\right)}{\left\|\nabla G\left(\mathbf{u}_{k}\right)\right\|}$,

- Calculate $\mathbf{u}_{k+1}=-\beta_{k} \alpha_{k}$,

- If $\left\|\mathbf{u}_{k+1}-\mathbf{u}_{k}\right\| \leq \varepsilon$ stop the calculation; otherwise set $k=$ $k+1$ and go to step 2 .

After convergence, it is possible to check that $G\left(\mathbf{u}_{k}\right)=0$.

Quapro is a linear quadratic optimization algorithm of ATOMS module of Scilab. Quapro algorithm uses a based decomposition method QR and Cholesky factorization of the Hessian matrix of the constraints to solve the optimization problem [71]. It aims at solving the following problem:

$$
\left\{\begin{array}{c}
f(\mathbf{x})=\frac{1}{2} \mathbf{x}^{T} \mathbf{H} \mathbf{x}+\mathbf{p}^{T} \mathbf{x} \\
\text { Subject to } \quad \mathbf{A x} \leq \mathbf{b} \\
{ }_{c_{\text {inf }, i} \leq x_{i} \leq c_{\text {sup }, i}, \quad 1 \leq i \leq n}
\end{array}\right.
$$

where $\mathbf{H}$ is an $n \times n$ symmetric matrix, while $\mathbf{p}$ and $\mathbf{b}$ are $n$-vectors.

The used Quapro algorithm can be summarized in the following steps:

- $i_{q p}=1$, allowable error $\varepsilon_{q p}=10^{-4}$. Definition of the matrix $D^{i q p}$ of signed distance between cylinders 1 and 2 ,

- Calculate the matrix $\mathbf{H}$ and the vector $\mathbf{p}$ associated to a selected point in the surface 2 ,

- Calculate the optimal fixed pitch and constraints related to $\mathbf{x}$ as:

$$
\begin{aligned}
p^{i q p} & =\left(\frac{1}{\text { length }(\mathbf{x})}-10 \varepsilon_{q p}\right) * \max \left(\varepsilon_{q p} ; \min \left(\left|D^{i q p}\right|\right)\right) \\
c_{\text {inf }, i} & =p^{i q p} \cdot\left(\begin{array}{c}
-1 \\
-1 \\
-0.1 \\
-0.1
\end{array}\right) \text { and } c_{\text {sup }, i}=p^{i q p} \cdot\left(\begin{array}{c}
1 \\
1 \\
0.1 \\
0.1
\end{array}\right) .
\end{aligned}
$$

- Minimize using Quapro $f(\mathbf{x})=\frac{-1}{2}\|\mathbf{x}\|^{2}$ subject to $\mathbf{A x} \leq$ b, $\quad c_{\text {inf }, i} \leq x_{i} \leq c_{\text {sup }, i}, \quad 1 \leq i \leq n$, a new vector $\mathbf{x}^{\text {new }}$ is obtained,
- Perform a displacement of surface 2 through $\mathbf{x}^{\text {new }}$,

- Calculate the new matrix of signed distance $D^{i q p+1}$ and update the vector including the sum of all the successive optimizations: $\mathbf{x}^{\text {opt }}=\mathbf{x}^{\text {opt }}+\mathbf{x}^{\text {new }}$,

- If $\min \left(D^{i q p+1}\right) \leq \varepsilon_{q p}$ or $\left\|\mathbf{x}^{\text {new }}\right\| \leq \varepsilon_{q p}$ stop the calculation; otherwise set $i_{q p}=i_{q p}+1$ and go to step 2 .

For the studied case of cylindrical pair joint, the algorithm converges in at most 150 iterations. Beyond this point, the joint is not mountable and there is interference between surfaces.

\section{References}

[1] Chen $H$, Jin S, Li Z, Lai X. A comprehensive study if three dimensional tolerance analysis methods. Comput Aided Des 2014;53:1-13.

[2] Dantan JY, Gayton N, Etienne A, Qureshi AJ, Dumas A. Mathematical issues in mechanical Tolerance analysis. In: 13e colloq. natl. aip primeca. Mont Dore. 2012.

[3] Chase KW, Greenwood WH. Design issues in mechanical Tolerance analysis. Manuf Rev 1987; 1:50-9.

[4] Qureshi AJ, Dantan JY, Sabri V, Beaucaire P, Gayton N. A statistical tolerance analysis approach for over-constrained mechanism based on optimisation and Monte-Carlo simulation. Comput Aided Des 2012;44:132-42.

[5] Ameta G. Statistical tolerance analysis and allocation for assemblies using Tolerance-maps [Ph.D. thesis]. Arizona State university; 2006.

[6] Nigam SD, Turner JU. Review of statistical approaches of tolerance analysis. Comput Aided Des 1995;27:6-15.

[7] Homri L, Teissandier D, Ballu A. Tolerance analysis by polytopes: Taking into account degrees of freedom with cap half-spaces. Comput Aided Des 2015;62:112-30.

[8] Pierce RS, Rosen D. A method for integrating form errors into tolerance analysis. J Mech Des 2007;130.

[9] Adragna PA, Faverlière H, Samper S, Pillet M. Statiscal assemblies with form errors - a 2D example. In: Ratcchev S, Koelmeijer S, editors. Micro-assmbly technol. appl. Boston: Springer; 2008. p. 23-33.

[10] Grandjean J, Ledoux Y, Samper S. On the role of form defects in assemblies subject to local deformations and mechanical loads. Int J Adv Manuf Technol 2013;65:1769-78.

[11] Schleich B, Anwer N, Mathieu L, Wartzack S. Contact and mobility simulation for mechanical assemblies based on skin model shapes. Ournal Comput Inf Sci Eng 2015;15.

[12] Bourdet P, Mathieu L, Lartigue C, Ballu A. The concept of the small displacement torsor in metrology. Ser Adv Math Appl Sci Adv Math Tools Metrol Ii 1996;40:110-22.

[13] Chase KW, Parkinson AR. A survey of research in the application of tolerance analysis to the design of mechanical assemblies. Res Eng Des 1991;3:23-37.

[14] Turner JU. Relative positionning of parts in assemblies using mathematical programming. Comput Aided Des 1990;22:394-400.

[15] Serre P, Rivière A, Clément A. Analysis of functional geometrical specifications Cachan (France): Kluwer academic publisher; 2001. p. 115-25.

[16] Morière S, Mailhé J, Linares JM, Sprauel JM. Assembly Method Comparison including Form Defect, in: 11th cirp int. conferance comput. aided toler. 2009

[17] Merkley K. Tolerance analysis of complaint assemblies [Ph.D. dissertation] Brigham Young University; 1998.

[18] Gupta S, Turner JU. Variational solid modeling for tolerance analysis. Comput Graph Appl Ieee 1993;13:64-74

[19] Franciosa P, Gerbino S, Patalano S. Simulation of variational compliant assemblies with shape errors based on morphing mesh approach. Int J Adv Manuf Technol 2011;53:47-61

[20] Ballu A, Mathieu L. Analysis of dimensional and geometrical specifications: standards and models. In: Proc. 3rd cirp semin. comput. aided toler. Cachan (France). 1993.

[21] Dantan JY, Ballu A, Mathieu L. Geometrical product specifications: model for product lifecycle. Comput Aided Des 2008;40:493-501. http://dx.doi.org/10, 1016/j.cad.2008.01.004.

[22] Dantan JY. Comparison of skin model representations and tooth contact analysis techniques for Gear Tolerance analysis. J Comput Inf Sci Eng 2015;15.

[23] Schleich B, Wartzack S. Approaches for the assembly simulation of skin model shapes. Comput Aided Des 2015;65:18-33.

[24] Schleich B, Anwer N, Mathieu L, Wartzack S. Skin model shapes: a new paradigm shift for geometric variations modelling in mechanical engineering. Comput Aided Des 2014;50:1-15.

[25] Huang W, Ceglarek D. Mode-based decomposition of part form error by discrete-cosine-transform with implementation to assembly and stamping system with compliant parts. Cirp Ann - Manuf Technol 2002;51:21-6.

[26] Lecompte J, Legoff O, Hascoet JY. Technological form defects identification using discrete cosine transform method. Int J Adv Manuf Technol 2010;51: 1033-44. 
[27] Raja J, Radhakrishnan V. Analysis and synthesis of surface profiles using fourier series. Int J Mach Tool Des Res 1977;17:245-51.

[28] Henke RP, Summerhays KD, Baldwin JM, Cassou RM, Brown CW. Methods for evaluation of systematic geometric deviations in machined parts and their relationships to process variables. Precis Eng 1999;23:273-92.

[29] Camelio JA, Hu SJ, Marin SP. Compliant assembly variation analysis using component geometric covariance. J Manuf Sci Eng 2004;126:355-60.

[30] Samper S, Formosa F. Form defects tolerancing by natural modes analysis. J Comput Inf Sci Eng 2006;7.

[31] Ballu A, Mathieu L, Legoff O. Representation of mechanical assemblies and specifications by graphs. In: Geom. toler. prod. ISTE-WILEY; 2010. p. 87-110.

[32] Dantan JY, Mathieu L, Ballu A, Martin P. Tolerance synthesis: quantifier notion and virtual boundary. Comput Aided Des 2005;37:231-40.

[33] Soderberg R, Lindkvist L. Automated seam variation and stability analysis for automotive body system. Cachan (France): Kluwer academic publisher; 2001. p. 255-64.

[34] Davidson JK, Mujezinovic A, Shah JJ. A new mathematical model for geometric tolerances as applied to round faces. ASME Trans J Mech Des 2002;124:609-22.

[35] Singh G, Ameta G, Davidson JK, Shah JJ. Worst-case tolerance analysis of a selfaligning coupling assembly using tolerance-maps. In: 11th cirp int. conferance comput. aided toler. Annecy (France). 2009.

[36] Dantan JY, Qureshi AJ. Worse case and statistical tolerance analysis based on quantified constraint satisfaction problems and monte carlo simulation. Comput Aided Des 2009;41:1-12.

[37] Dumas A, Dantan JY, Gayton N. Impact of a behavior model linearization strategy on the tolerance analysis of over-constrained mechanisms. Comput Aided Des 2015;62:152-63.

[38] Lê HN, Ledoux Y, Ballu A. Experimental and theoretical investigations of mechanical joints with form defects. J Comput Inf Sci Eng 2014;14.

[39] Cho N, Tu JF. Quantitative circularity tolerance analysis and design for 2D precision assemblies. Int J Mach Tools Manuf 2002;42:1391-401.

[40] Giordano M, Duret D. Clearance space and deviation space. In: 3rd cirp semin. comput. aided toler. Cachan (France). 1993. p. 179-96.

[41] Fleming AD. Analysis of uncertainties and geometric tolerances in assemblies of parts [Ph.D. Thesis]. University of Edinburgh; 1987.

[42] Fleming AD. Geometric relationships between toleranced features. Artificial Intelligence 1988;37:403-12. http://dx.doi.org/10.1016/0004-3702(88)900628.

[43] Zou Z, Morse EP. A gap-based approach to capture fitting conditions for mechanical assembly. Comput Aided Des 2004;36:691-700.

[44] Bhide S, Ameta G, Shah JJ, Davidson JK. Tolerance-Maps applied to the straightness and orientation of an axis. In: Cirp models comput. aided toler. des. manufactering. 2005. p. 45-54.

[45] Shah JJ, Ameta G, Shen Z, Davidson JK. Navigating the tolerance analysis maze. Comput Aided Des Appl. 2007;4:705-18.

[46] Nigam SD, Turner JU. Review of statistical approaches of tolerance analysis. Comput Aided Des 1995;27:6-15.

[47] Beaucaire P, Gayton N, Duc E, Dantan JY. Statistical tolerance analysis of overconstrained mechanisms with gaps using system reliability methods. Comput Aided Des 2013;45:47-55.

[48] Guilford J, Turner JU. Advanced tolerance analysis and synthesis for geometric tolerances. In: Proc. int. forum dimens. toler. metrol. 1993. p. 187-98.

[49] Zhang C, Luo J, Wang B. Statistical tolerance synthesis using distribution function zones. Int J Prod Res 1999;37:3995-4006.

[50] Whitney DE, Gilbert OL, Jastrzebski M. Representation of geometric variations using matrix transforms for statistical tolerance analysis in assemblies. Res Eng Des 1994;6:191-210.
[51] Johannesson H, Soderberg R. Structure and matrix models for tolerance analysis from configuration to detail design. Res Eng Des 2000;12:112-25.

[52] Chase KW, Magleby SP, Glancy CG. Tolerance analysis of 2-D and 3-D mechanical assemblies with small kinematic adjustments. In: Zhang HC, editor. Adv. toler. tech. Wiley; 1997.

[53] Laperrière L, Lafond P. Tolerance Analysis and synthesis using virtual joints. In: Ensheded - Netherland, 1999. p. 405-14.

[54] Teissandier D, Delos V, Couétard Y. Operations on polytopes: Application to tolerance analysis. Enschede (Netherlands): Kluwer academic publisher; 1999. p. 425-33.

[55] Germain F, Denimal D, Giordano M. A method for three dimensional tolerance analysis and synthesis applied to complex and precise assemblies. Proc Int Fed Inf Process 2008;260:55-65.

[56] Mansuy M, Giordano M, Hernandez P. A new calculation method for the worst case tolerance analysis and synthesis in stack-type assemblies. Comput Aided Des 2011;43:1118-25.

[57] Bjorke O. Computer-aided tolerancing. 2nd ed. New-York USA: ASME Press; 1989.

[58] Dumas A, Dantan JY, Gayton N. Impact of a behavior model linearization strategy on the tolerance analysis of over-constrained mechanisms. Comput Aided Des 2015;62:152-63.

[59] Strohmer T, Tanner J. Implementations of Shannon's sampling theorem, a time-frequency approach. Sampl Theory Signal Image Process 2005;4: $1-17$.

[60] Formosa F, Samper S. Modal expression of form defects. In: Davidson JK, editor. Models comput. aided toler. des. manuf. Springer; 2007. p. 13-22.

[61] Pierce RS, Rosen D. Simulation of mating between monanalytic surfaces using a mathematical programing formulation. J Comput Inf Sci Eng 2007;7: 314-21.

[62] Huang QH, Flöry S, Gelfand N, Hofer M, Pottman H. Reassembling fractured objects by geometric matching. ACM Trans Graph 2006;25:569-78.

[63] Samper S, Adragna PA, Favreliere H, Pillet M. Modeling of 2D and 3D assemblies taking into account form errors of plane surfaces. J Comput Inf Sci Eng 2009;9(n.d.)

[64] Hasofer AM, Lind NC. Exact and invariant second-moment code format. J Eng Mech Div 1974;100:111-21.

[65] Rackwitz R, Fiessler B. Structural reliability under combined random load sequences. Comput Struct 1978;9:489-94.

[66] Periçaro GA, Santos SR, Ribeiro AA, Matioli LC. HLRF-BFGS optimization algorithm for structural reliability. Appl Math Model 2015;39:2025-35.

[67] Zhang Y, Kiureghian AD. Finite element reliability methods for inelastic structures. Berkeley: Department of Civil and Environmental Engineering, University of California; 1997.

[68] Nemirovski A. Interior point polynomial time methods in convvex programming. Georgia Institute of Technology School of Industrial and Systems Engineering; 2004

[69] Nelder J. A simplex method for function minimization. Comput J 1965;7: 308-13.

[70] Baudin M. Nelder-Mead user's manual. 2010.

[71] Casas E, Pola C. An algorithm for indefinite quadratic programming based on a partial cholesky factorization. Rev Fr Autom Inform Rech Oper 1993;7: 401-26.

[72] ISO286-1, ISO system of limits and fits - Part 1: Bases of tolerances, deviations and fits. 1988.

[73] Clément A, Rivière A, Serré P, Valade C. The TTRS: 13 constraints for dimensioning and tolerancing. Toronto (Canada): Chapman \& Hall; 1998, p. 122-9. 